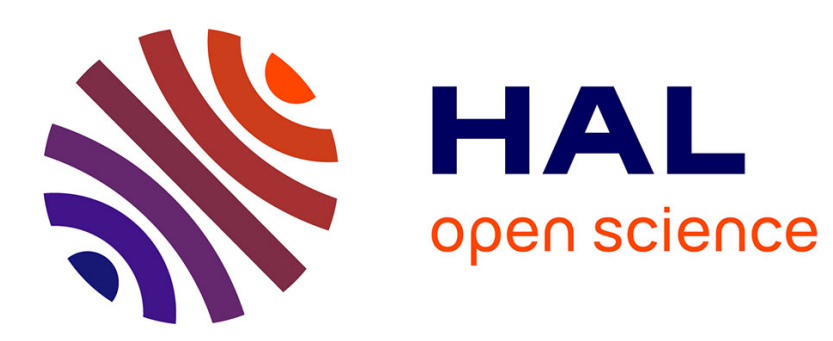

\title{
Accurate absorbing boundary conditions for two-dimensional peridynamics
}

Gang Pang, Songsong Ji, Xavier Antoine

\section{To cite this version:}

Gang Pang, Songsong Ji, Xavier Antoine. Accurate absorbing boundary conditions for twodimensional peridynamics. Journal of Computational Physics, 2022, 466 (1), pp.111351. 10.2139/ssrn.3952372 . hal-03399896

\section{HAL Id: hal-03399896 https://hal.science/hal-03399896}

Submitted on 24 Oct 2021

HAL is a multi-disciplinary open access archive for the deposit and dissemination of scientific research documents, whether they are published or not. The documents may come from teaching and research institutions in France or abroad, or from public or private research centers.
L'archive ouverte pluridisciplinaire HAL, est destinée au dépôt et à la diffusion de documents scientifiques de niveau recherche, publiés ou non, émanant des établissements d'enseignement et de recherche français ou étrangers, des laboratoires publics ou privés. 


\title{
Accurate absorbing boundary conditions for two-dimensional peridynamics
}

\author{
Gang PANG ${ }^{\mathrm{a}}$, Songsong $\mathrm{JI}^{\mathrm{b}}$, Xavier ANTOINE ${ }^{\mathrm{c}, *}$ \\ ${ }^{a}$ School of Mathematical Science, Beihang University, Beijing 102206, China \\ ${ }^{b}$ HEDPS, CAPT, and LTCS, College of Engineering, Peking University, Beijing 100871, China \\ ${ }^{c}$ Université de Lorraine, CNRS, Inria, IECL, F-54506 Vandoeuvre-lès-Nancy Cedex, France
}

\begin{abstract}
The aim of this paper is to construct accurate absorbing boundary conditions (ABCs) for the two-dimensional peridynamics equation of motion which describes nonlocal phenomena arising in continuum mechanics based on integrodifferential equations. To this end, a full discretization of the system is used based on a Crank-Nicolson scheme in time and an asymptotically compatible scheme in space. Recursive relations for the Green's functions are then derived and numerically used to evaluate the nonlocal ABCs. In particular, these absorbing boundary conditions solve the corner reflection problem with high precision. The stability of the complete fully discretized scheme is stated and numerical examples are finally reported to demonstrate the validity of the resulting
\end{abstract} ABCs.

Keywords: Two-dimensional peridynamics equation of motion; integrodifferential operator; absorbing boundary condition; corner reflection; discrete Green's function; asymptotically compatible discretization

\section{Contents}

1 Introduction 2

2 Absorbing boundary conditions for the fully discretized peridynamics 3

2.1 Time and space discretizations of the two-dimensional peridynamics system . . . . . 3

2.2 Construction of the absorbing boundary conditions for the fully discretized peridy-

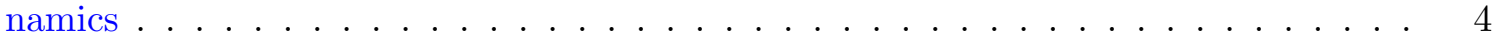

2.3 Computation of the function $\left\{g_{j, \ell}^{n}\right\}_{n}$ and Green's function $\left\{\mathbb{C}^{n}\right\}_{n} \ldots \ldots 11$

2.4 Numerical algorithm for the fully discretized peridynamics system with ABC . . . 14

3 Properties of the kernel $\left\{\mathbb{C}^{n}\right\}_{n}$ and stability analysis $\quad 14$

3.1 Properties of the kernel $\left\{\mathbb{C}^{n}\right\}_{n} \ldots \ldots \ldots \ldots \ldots \ldots \ldots$

3.2 Stability analysis . . . . . . . . . . . . . . . . . . 19

4 Numerical example $\quad 21$

5 Conclusion $\quad 21$

*Author for correspondence: xavier.antoine@univ-lorraine.fr 


\section{Introduction}

A wide variety of nonlocal models has emerged over the last past years in many areas of physics and engineering [9]. In particular, the recent nonlocal peridynamic theory, introduced by Silling $[9,31]$, has been developed to reformulate the classical local equations of continuum mechanics to deal with material fracture problems [15, 21, 26, 27, 38], deformations [31], damage, cracking and wave propagation of peridynamics in unbounded media [36, 37]. Since analytical solutions are usually not available for general peridynamics models $[25,36]$, numerical simulations are strongly needed to investigate the behavior of the solutions [7, 22, 23, 24]. In the present paper, we consider the two-dimensional peridynamics equation of motion (with constant density) set in an unbounded medium which consists in searching for the unknown displacement field $u$ solution to

$$
\left\{\begin{array}{l}
u_{t t}(x, y, t)=\mathcal{L}_{\delta} u(x, y, t)+f(x, y), \quad(x, y) \in \mathbb{R}^{2}, \quad t \in(0, T] \\
u(x, y, 0)=\phi^{0}(x, y), \quad(x, y) \in \mathbb{R}^{2}, \\
u_{t}(x, y, 0)=\phi^{1}(x, y), \quad(x, y) \in \mathbb{R}^{2}, \\
\lim _{|x|^{2}+|y|^{2} \rightarrow+\infty} u(x, y, t)=0, \quad t \in(0, T],
\end{array}\right.
$$

where $\phi^{0,1}$ are two given initial data, $f$ is the body force and the maximal time of computation is $T$. The nonlocal operator $\mathcal{L}_{\delta}$ appearing in (1) is given by $[9,11]$

$$
\mathcal{L}_{\delta} u(x, y, t)=\int_{\mathbb{R}^{2}} \gamma_{\delta}\left(x^{\prime}, y^{\prime}\right)\left(u\left(x+x^{\prime}, y+y^{\prime}, t\right)-u(x, y, t)\right) d x^{\prime} d y^{\prime}
$$

where the interaction kernel function $\gamma_{\delta}$ has the following properties

- $\gamma_{\delta}$ is a radial function according to $r^{\prime}=\sqrt{\left(x^{\prime}\right)^{2}+\left(y^{\prime}\right)^{2}}: \gamma_{\delta}\left(x^{\prime}, y^{\prime}\right)=\gamma_{\delta}\left(r^{\prime}\right)$;

- $\gamma_{\delta}$ is positive: $\gamma_{\delta}\left(r^{\prime}\right) \geq 0$;

- $\gamma_{\delta}$ has a finite horizon $\delta$, i.e. $\gamma_{\delta}\left(r^{\prime}\right)=0$, for $r^{\prime}>\delta>0$.

For the one-dimensional case [9], the integrodifferential operator $\mathcal{L}_{\delta}$ generalizes the fractional Laplacian $\left(-\partial_{x}^{2}\right)^{s / 2}$, with $1 \leq s \leq 2$. Generally speaking, nonlocal PDEs [8, 9] describe long-range interactions in a given system through the kernel function $\gamma_{\delta}$.

The method of absorbing/artificial boundary conditions (ABCs) (also sometimes called transparent or non-reflecting boundary conditions) is used in the present paper to reformulate the unbounded problem (1) into an initial boundary-value problem set in a square finite computational domain such that the waves striking the fictitious boundary could be efficiently annihilated. Since a few decades, many contributions (see e.g. $[1,3,6,13,14,16,17,18,33]$ ) were devoted to the design and implementation of accurate ABCs for integer order local PDEs, and in particular for wave-like equations. In the case of nonlocal PDEs, much less works on ABCs are available in the literature. In [12], the authors propose an $\mathrm{ABC}$ for the one-dimensional nonlocal wave equation. For the case of one-dimensional nonlocal Schrödinger equations, we refer to $[28,39]$ for the boundary treatment and numerical analysis of ABCs. DtN-based boundary operators are developed in [40, 41] for onedimensional nonlocal heat equations. The absorbing layers/PML approach is investigated for the one-dimensional heat and Schrödinger equations involving fractional operators in [5, 20]. A general PML approach is introduced in [2] for fractional PDEs, and completed with techniques in [4] which are more specifically devoted to the fractional Laplacian. Some efficient local in-time ABCs are proposed for the one-dimensional peridynamics case in [32, 35]. However, these algorithms meet difficulties when dealing with the corner problem for two- and three-dimensional domains. For two-dimensional nonlocal PDEs, ABCs are constructed in [10] but without stability analysis. In 
general, much less works dedicated to ABCs for two-dimensional spatially nonlocal and fractional PDE models are available. A crucial difficulty when dealing with these nonlocal problems is to compute the Green's functions accurately [34]. In the present work, we solve this problem by using techniques developed in [19, 29, 30], where the Green's functions for both the semi-discretized heat and Schrödinger equations in a two-dimensional domain are computed accurately. The corresponding boundary conditions solve the problem of corner reflection for the two-dimensional peridynamics model with high precision.

The aim of the present paper is (i) to construct ABCs for a fully-discretized version of (1), (ii) to develop a stability analysis of the resulting scheme and (iii) to confirm the accuracy of the approach through numerical examples. To this end, a Crank-Nicolson time discretization and a spatial asymptotically compatible scheme are applied to (1) to obtain a full discretization in Subsection 2.1. Then, the exact ABC for the fully discretized version of (1) is extracted in Subsection 2.2. In addition, an efficient recursive algorithm is proposed in Subsection 2.3 to compute with high accuracy the discrete Green's functions which are involved in the construction of the ABCs in Subsection 2.4. In Section 3, the stability analysis of the scheme with ABCs is developed. Numerical examples are reported in Section 4 to confirm the effectiveness of the ABCs. Finally, we end by a conclusion in Section 5.

\section{Absorbing boundary conditions for the fully discretized peridynamics}

To construct the accurate absorbing boundary conditions for the two-dimensional peridynamics system (1), we first derive its fully discretized version in Subsection 2.1. Next, the corresponding ABCs are proposed in Subsections 2.2 and 2.3, before providing the full scheme in Subsection 2.4.

\subsection{Time and space discretizations of the two-dimensional peridynamics system}

The Asymptotic Compatibility (AC) discretization scheme derived in [12] is used to approximate the integrodifferential operator $\mathcal{L}_{\delta}$ given by (2). The square computational domain $\left[-x_{J}, x_{J}\right] \times$ $\left[-x_{J}, x_{J}\right]$ is discretized by using $(2 J+1) \times(2 J+1)$ grid points $\left(x_{j}, y_{\ell}\right)=(j h, \ell h),-J \leq j, \ell \leq J$, for the uniform spatial meshsize $h:=x_{J} / J>0$. The piecewise multilinear basis function $\phi_{j, \ell}(x, y)$ is introduced such that $\phi_{j, \ell}\left(x_{m}, y_{k}\right)=\delta_{j, \ell}^{m, k}$, where we define the Krönecker symbol $\delta_{j, \ell}^{k, m}$, for $(k, m) \in \mathbb{Z}^{2}$ given, such that: $\delta_{j, \ell}^{k, m}=0$ for $(j, \ell) \neq(k, m)$ and $\delta_{j, \ell}^{k, m}=1$, otherwise. Then, by considering an integer $K \geq 0$, the AC discretization of $\mathcal{L}_{\delta}$ is given by: for $(j, \ell) \in \mathbb{Z}^{2}$

$$
\begin{aligned}
& \mathcal{L}_{\delta} u\left(x_{j}, y_{\ell}\right) \approx \\
& \sum_{k, m=0}^{K} b_{k, m}\left(u\left(x_{j+k}, y_{\ell+m}\right)+u\left(x_{j+k}, y_{\ell-m}\right)+u\left(x_{j-k}, y_{\ell+m}\right)+u\left(x_{j-k}, y_{\ell-m}\right)-4 u\left(x_{j}, y_{\ell}\right)\right),
\end{aligned}
$$

where the real-valued coefficients $b_{k, m}$ are such that

$$
b_{k, m}:= \begin{cases}\frac{k+m}{h\left(k^{2}+m^{2}\right)} \iint_{B^{+}(0, \delta)} \phi_{k, m}(x, y) \gamma_{\delta}\left(\sqrt{x^{2}+y^{2}}\right) \frac{x^{2}+y^{2}}{x+y} d x d y, & (k, m) \neq(0,0), \\ 0, & (k, m)=(0,0),\end{cases}
$$

$B^{+}(0, \delta)$ being the first quadrant of the disc centered at the origin with radius $\delta$. It is clear that $b_{k, m}=b_{m, k}$.

For a sequence $u=\left\{u^{n}\right\}_{n=0}^{\infty}$ such that $\sum_{n=0}^{\infty}\left|u^{n}\right|^{2}<\infty$, the operators $S^{ \pm}$are defined by: $S^{ \pm} u=\left\{u^{n \pm 1}\right\}_{n=0}^{\infty}$, setting $u^{-1}=0$. The average operators $E^{ \pm}$and difference quotient operators $D_{\tau}^{ \pm}$with uniform time step $\tau$ are given by $E^{ \pm}=\left(S^{ \pm}+I\right) / 2$ and $D_{\tau}^{ \pm}= \pm\left(S^{ \pm}-I\right) / \tau$, respectively. 


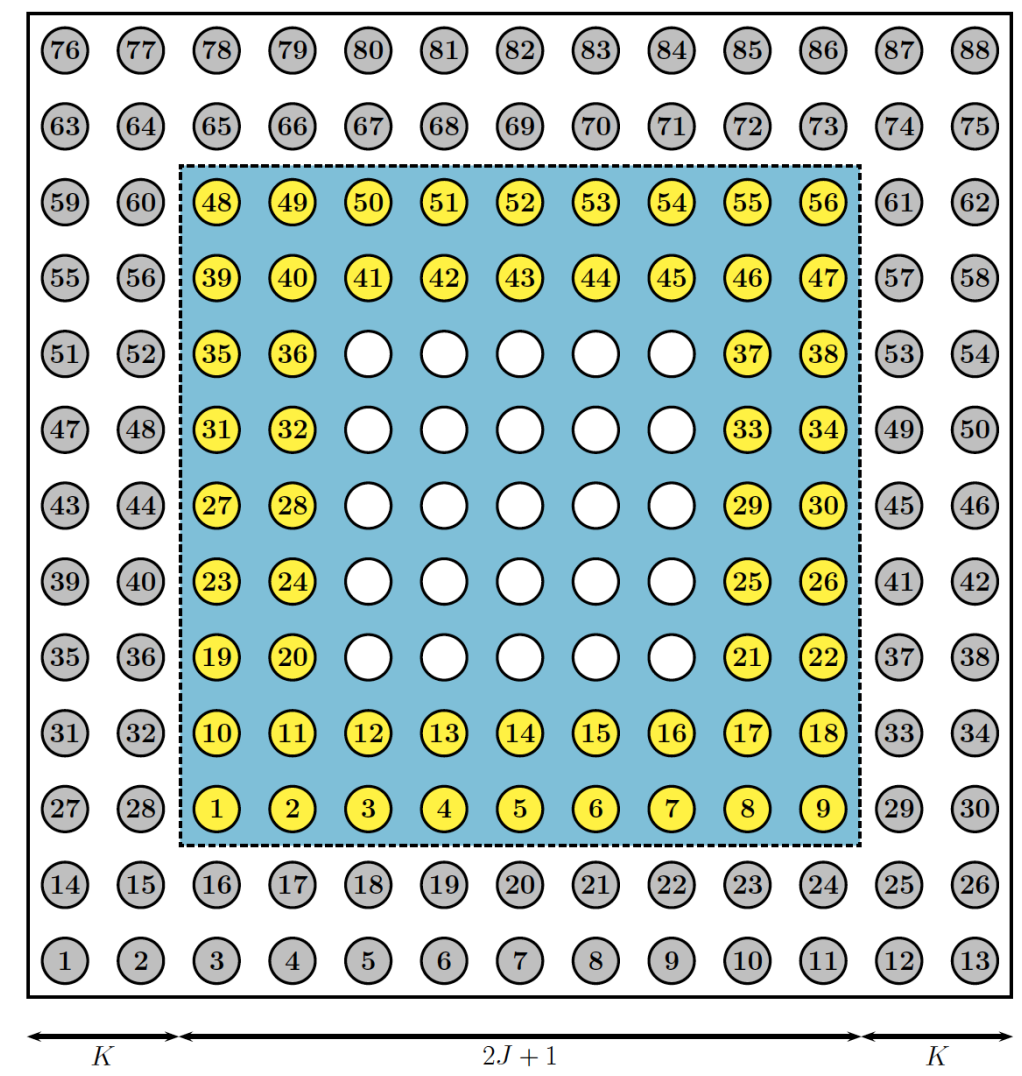

Figure 1: Labelling of the interior and exterior layers nodes $(J=4$ and $K=2)$.

The following symbols are used: $S^{ \pm} u^{n}=\left(S^{ \pm} u\right)^{n}, E^{ \pm} u^{n}=\left(E^{ \pm} u\right)^{n}$ and $D_{\tau}^{ \pm} u^{n}=\left(D_{\tau}^{ \pm} u\right)^{n}$. Let us introduce the coefficients:

$$
a_{k, m}=b_{|k|,|m|}, \quad(k, m) \neq(0,0), \quad a_{0,0}=-\sum_{-K \leq k, m \leq K,(k, m) \neq(0,0)} b_{k, m} .
$$

Based on the Crank-Nicolson $(\mathrm{CN})$ time scheme at time $t_{n}=n \tau$ and the AC spatial discretization (3), we obtain the fully discretized peridynamics system (called CNAC) approximating (1)(for $f=0)$

$$
D_{\tau}^{-} D_{\tau}^{+} u_{j, \ell}^{n}=E^{-} E^{+} L_{\delta, h} u_{j, \ell}^{n}:=\sum_{k, m=-K}^{K} a_{k, m} E^{-} E^{+} u_{j+k, \ell+m}^{n}, \quad \text { for } j, \ell \in \mathbb{Z},
$$

where $u_{j, \ell}^{n}$ denotes the numerical approximation of $u\left(x_{j}, y_{\ell}, t_{n}\right)$.

\subsection{Construction of the absorbing boundary conditions for the fully discretized peridynamics}

We propose to derive the exact absorbing boundary conditions for general compactly supported initial data $\phi^{0}$ and $\phi^{1}$. For convenience, we introduce the notation $\mathcal{R}_{L}(m, n)$ to denote a rectangular box in $\mathbb{Z}^{2}$ such that $\mathcal{R}_{L}(m, n)=\{(p, q):|p-m| \leq L,|q-n| \leq L\}$. The finite rectangular computational domain can be chosen as $\mathcal{R}_{J}(0,0)$, with size $(2 J+1) \times(2 J+1)$ (blue square domain in Figure 1 for the specific case $J=4$ and $K=2$ ), such that $u_{j, \ell}^{n}$ is located at $(j, \ell)$, with $j=$ $-J, \ldots, J$ and $\ell=-J, \ldots, J$. Therefore, the white interior points correspond to $\mathcal{R}_{J-K}(0,0)$, the yellow interior layer points to $\mathcal{R}_{J}(0,0) / \mathcal{R}_{J-K}(0,0)$ and and finally the gray exterior layer points to 
$\mathcal{R}_{J+K}(0,0) / \mathcal{R}_{J}(0,0)$. Let us denote by $\bmod (a, b)$ the modulo function which provides the remainder of the division of $a$ by $b$. Then, we define the symbol $\nu(k), 1 \leq k \leq 4 K(2 J+1-K)$, to denote the location of the $k$-th interior layer node (yellow node in Figure 1) such that

- For $1 \leq k \leq(2 J+1) K$ :

1. If $\bmod (k, 2 J+1) \neq 0$ :

$$
\nu(k)=\left(-J+\frac{k-\bmod (k, 2 J+1)}{2 J+1},-J+\bmod (k, 2 J+1)-1\right)
$$

2. If $\bmod (k, 2 J+1)=0$ :

$$
\nu(k)=\left(-J+\frac{k-\bmod (k, 2 J+1)}{2 J+1}-1, J\right)
$$

- For $(2 J+1) K+1 \leq k \leq(2 J+1) K+(2 J+1-2 K) 2 K$ :

Set: $k_{1}=k-(2 J+1) K-1$

1. If $\bmod \left(k_{1}, 2 K\right) \neq 0$ :

(a) If $\bmod \left(k_{1}, 2 K\right) \leq K$ :

$$
\nu(k)=\left(-J+K+\frac{k-\bmod (k, 2 K)}{2 K},-J+\bmod (k, 2 K)-1\right)
$$

(b) If $\bmod \left(k_{1}, 2 K\right) \geq K+1$ :

$$
\nu(k)=\left(-J+K+\frac{k-\bmod (k, 2 K)}{2 K}, J-(2 K-\bmod (k, 2 K))+1\right)
$$

2. If $\bmod \left(k_{1}, 2 K\right)=0$ :

$$
\nu(k)=\left(-J+K+\frac{k_{1}-\bmod \left(k_{1}, 4 K\right)}{4 K}-1, J\right)
$$

- For $(2 J+1) K+(2 J+1-4 K) 2 K+1 \leq k \leq J^{2}-(J-2 K)^{2}$ :

Set $k_{1}=k-(2 J+1) K-(2 J+1-2 K) 2 K$

1. If $\bmod \left(k_{1}, 2 J+1\right) \neq 0$ :

$$
\nu(k)=\left(J-K+1+\frac{k_{1}-\bmod \left(k_{1}, 2 J+1\right)}{2 J+1},-J+\bmod \left(k_{1}, 2 J+1\right)-1\right)
$$

2. If $\bmod \left(k_{1}, 2 J+1\right)=0$ :

$$
\nu(k)=\left(J-K+1+\frac{k_{1}-\bmod \left(k_{1}, 2 J+1\right)}{2 J+1}-1, J\right)
$$

The location of the $m$-th exterior layer node (black node in Figure 1 ) is labelled by the symbol $\mu(m)$ in the same way. The number of interior layer nodes is equal to $(2 J+1)^{2}-(2 J+1-2 K)^{2}=$ $4 K(2 J+1-K)$. We use $\mathcal{B}_{\text {Int }}$ to denote the set consisting of the locations of interior layer nodes, namely,

$$
\mathcal{B}_{\text {Int }}=\{\nu(k): 1 \leq k \leq 4 K(2 J+1-K)\}
$$


with cardinal $\left|\mathcal{B}_{\text {Int }}\right|=4 K(2 J+1-K)$. We also define: $\mathcal{B}_{\text {Ext }}=\{\mu(k): 1 \leq k \leq 4 K(2 J+1+K)\}$, with $\left|\mathcal{B}_{\text {Ext }}\right|=4 K(2 J+1+K)$. The notation $(\mathbf{I}[u])^{n}:=\left[u_{\nu(1)}^{n}, \cdots, u_{\nu\left(\left|\mathcal{B}_{\text {Int }}\right|\right)}^{n}\right]^{T}$ is used for the interior layer vector such that $u_{\nu(k)}^{n}$ is located at the point $\nu(k)$ in $\mathcal{B}_{\text {Int }}$ (with $\mathbb{M}^{T}$ the transpose of any complex-valued matrix $\mathbb{M})$. Similarly, the exterior layer vector is $(\mathbf{E}[u])^{n}:=\left[u_{\mu(1)}^{n}, \cdots, u_{\mu\left(\left|\mathcal{B}_{\text {Ext }}\right|\right)}\right]^{T}$.

We define the $\mathcal{Z}$-transform of a given complex-valued sequence $\left(f^{n}\right)_{n \in \mathbb{N}}$ by

$$
\widehat{f}(z):=\sum_{k=0}^{\infty} f^{n} z^{-n} .
$$

For a sequence of $M \times M$ matrices $\left(\mathbb{A}_{n}\right)_{n \in \mathbb{N}}$ and for a sequence of vectors $\left(\mathbf{v}_{n}\right)_{n \in \mathbb{N}} \in \mathbb{C}^{M}$, the associated $\mathcal{Z}$-transforms are:

$$
\widehat{\mathbb{A}}(z):=\sum_{n=0}^{\infty} \mathbb{A}^{n} z^{-n} \quad \text { and } \quad \widehat{\mathbf{v}}(z):=\sum_{n=0}^{\infty} \mathbf{v}^{n} z^{-n} .
$$

In addition, the convolution $\star$ of two sequences $\left\{g^{n}\right\}_{n}$ and $\left\{f^{n}\right\}_{n}$ is such that

$$
(f \star g)^{n}:=\sum_{r=0}^{n} g^{n-r} f^{n},
$$

while for two sequences of matrices $\left\{\mathbb{A}^{n}\right\}_{n}$ and $\left\{\mathbb{B}^{n}\right\}_{n}$ of size $M \times M$ we have:

$$
(\mathbb{A} \star \mathbb{B})^{n}:=\sum_{r=0}^{n} \mathbb{A}^{n-r} \mathbb{B}^{r} .
$$

Finally, we introduce the function

$$
\rho_{\text {Int }}(z, x, y):=\left(\frac{z-1}{\tau}\right)^{2}-\left(\frac{z+1}{2}\right)^{2} A_{\text {Int }}(x, y),
$$

with (setting $\mathrm{i}=\sqrt{-1}$ )

$$
A_{\text {Int }}(x, y):=\sum_{k, m=-K}^{K} a_{k, m} e^{-\mathrm{i} k x} e^{-\mathrm{i} m y} .
$$

From the assumption on the compact support of $\phi^{0}$, the discrete initial data $u_{j, \ell}^{0}$ is zero outside the selected computational domain, including its interior layer (i.e. compactly supported in the domain consisting of the white points in Figure 1). In this setting, we can extract the ABCs to complete the CNAC discrete system (5), restricted to indices $(j, \ell) \in \mathcal{B}_{\text {Int }}$. To this end, a relation between the unknowns $u_{\mu(j)}^{n}\left(1 \leq j \leq\left|\mathcal{B}_{\text {Ext }}\right|\right)$ in the exterior layer (black dots in Figure 1$)$ and the values $u_{\nu(j)}^{n}\left(1 \leq j \leq\left|\mathcal{B}_{\text {Int }}\right|\right)$ in the interior layer (yellow nodes) in the computational domain is derived in Theorem 1. This relation plays the role of an ABC.

Theorem 1. Under the above setting, the $A B C$ for the discretized peridynamics equation (5) is

$$
u_{\mu(j)}^{n}=\sum_{k=1}^{\left|\mathcal{B}_{\text {Int }}\right|}\left(\mathbb{C}_{j, k} \star u_{\nu(k)}\right)^{n}, \quad \text { for } 1 \leq j \leq\left|\mathcal{B}_{\text {Ext }}\right|,
$$


for $n \in \mathbb{N}$. Here, the sequence of matrices $\left\{\mathbb{C}^{n}\right\}_{n}$ of size $\left|\mathcal{B}_{\text {Ext }}\right| \times\left|\mathcal{B}_{\text {Int }}\right|$ is given through the relation: $\widehat{\mathbb{C}}(z)=\widehat{\mathbb{B}}(z) \widehat{\mathbb{A}}^{-1}(z)$, where $\mathbb{B}_{j, k}^{n}=f_{|\mu(j)-\nu(k)|}^{n}$, for $1 \leq j \leq\left|\mathcal{B}_{\text {Ext }}\right|, 1 \leq k \leq\left|\mathcal{B}_{\text {Int }}\right|$, and $\mathbb{A}_{j, k}^{n}=$ $f_{|\nu(j)-\nu(k)|}^{n}$, for $1 \leq j, k \leq\left|\mathcal{B}_{\text {Int }}\right|$, with

$$
\hat{f}_{j, \ell}(z)=\frac{\hat{g}_{j, \ell}(z)}{\hat{g}_{0,0}(z)}
$$

and

$$
\hat{g}_{j, \ell}(z)=\frac{1}{4 \pi^{2}} \int_{0}^{2 \pi} \int_{0}^{2 \pi}\left(\rho_{\text {Int }}(z, x, y)\right)^{-1} e^{-\mathrm{i} j x-\mathrm{i} \ell y} d x d y .
$$

In addition, the notation $|(m, n)|$ represents $(|m|,|n|)$.

To prove Theorem 1, we first need to state the following Lemma.

Lemma 1. The functions $\widehat{f}_{j, \ell}(z)$, for $(j, \ell) \in \mathbb{Z}^{2}$, satisfy the following equation

$$
\left(\frac{z-1}{\tau}\right)^{2} \widehat{f}_{j, \ell}(z)=\left(\frac{z+1}{2}\right)^{2} \sum_{k, m=-K}^{K} a_{k, m} \widehat{f}_{j+k, \ell+m}(z)+\frac{\delta_{j, \ell}^{0,0}}{\widehat{g}_{0,0}(z)} .
$$

Proof of Lemma 1. Let $j, \ell \in \mathbb{Z}$. A simple calculation based on (8)-(9) leads to

$$
\begin{aligned}
& \sum_{k, m=-K}^{K} a_{k, m} \widehat{f}_{j+k, \ell+m}(z)=\frac{1}{4 \pi^{2} \widehat{g}_{0,0}(z)} \int_{0}^{2 \pi} \int_{0}^{2 \pi} \rho_{\text {Int }}^{-1}(z, x, y) A_{\text {Int }}(x, y) e^{-\mathrm{i} j x-\mathrm{i} \ell y} d x d y \\
= & \frac{4}{(z+1)^{2} \widehat{g}_{0,0}(z)}\left(-\frac{1}{4 \pi^{2}} \int_{0}^{2 \pi} \int_{0}^{2 \pi} e^{-\mathrm{i} j x-\mathrm{i} \ell y} d x d y+\left(\frac{z-1}{\tau}\right)^{2} \widehat{g}_{j, \ell}\right) \\
= & \frac{4}{(z+1)^{2} \widehat{g}_{0,0}(z)}\left[\left(\frac{z-1}{\tau}\right)^{2} \widehat{g}_{j, \ell}(z)-\delta_{j, \ell}^{0,0}\right]
\end{aligned}
$$

which provides (10).

Let us now prove the following result based on Lemma 1.

Lemma 2. Let us set $N \tau=T$. We assume that the kernel functions $\left\{f_{j, \ell}^{n}\right\}_{n \in \mathbb{N}}$ satisfy (8)-(9), for $(j, \ell) \in \mathbb{Z}^{2}$. We consider $\left|\mathcal{B}_{\text {Int }}\right|$ given sequences $\left\{v_{\nu(k)}^{n}\right\}_{n \in \mathbb{N}}$, for $1 \leq k \leq\left|\mathcal{B}_{\text {Int }}\right|$, such that $v_{\nu(k)}^{0}=0$, $v_{\nu(k)}^{1}=0$ and $v_{\nu(k)}^{n}=0$, with $n \geq N+1$. If we define

$$
u_{j, \ell}^{n}=\sum_{k=1}^{\left|\mathcal{B}_{\text {Int }}\right|}\left(f_{(j, \ell)-\nu(k)} \star v_{\nu(k)}\right)^{n}, \quad \text { for }(j, \ell) \in \mathbb{Z}^{2} / \mathcal{R}_{J-K}(0,0),
$$

then, for any $(j, \ell) \in \mathbb{Z}^{2} / \mathcal{R}_{J}(0,0)$, we have

$$
D_{\tau}^{-} D_{\tau}^{+} u_{j, \ell}^{n}=E^{-} E^{+} L_{\delta, h} u_{j, \ell}^{n},
$$

with $u_{j, \ell}^{0}=0$ and $u_{j, \ell}^{1}=0$.

Proof of Lemma 2. It is clear that $\widehat{v}_{\nu(k)}(z)$ exists for $1 \leq k \leq\left|\mathcal{B}_{\text {Int }}\right|$. From (11), we obtain

$$
\widehat{u}_{j, \ell}(z)=\sum_{k=1}^{\left|\mathcal{B}_{\text {Int }}\right|} \widehat{f}_{(j, \ell)-\nu(k)}(z) \widehat{v}_{\nu(k)}(z), \quad \text { for }(j, \ell) \in \mathbb{Z}^{2} / \mathcal{R}_{J-K}(0,0) .
$$


For $(j, \ell) \in \mathbb{Z}^{2} / \mathcal{R}_{J}(0,0)$, we have: $(j, \ell)-\nu(k) \neq(0,0)$, with $1 \leq k \leq\left|\mathcal{B}_{\text {Int }}\right|$, and $\delta_{(j, \ell)-\nu(k)}^{0,0}=0$. Thus, from (10) and (13), the function $\widehat{u}_{j, \ell}(z)$ satisfies

$$
\begin{aligned}
& \left(\frac{z-1}{\tau}\right)^{2} \hat{u}_{j, \ell}(z)=\sum_{k=1}^{\left|\mathcal{B}_{\text {Int }}\right|}\left(\frac{z-1}{\tau}\right)^{2} \hat{f}_{(j, \ell)-\nu(k)}(z) \hat{v}_{\nu(k)}(z) \\
& =\left(\frac{z+1}{2}\right)^{2}\left[\sum_{k=1}^{\left|\mathcal{B}_{\text {Int }}\right|} \sum_{-K \leq p, q \leq K} a_{p, q} \hat{f}_{(j, \ell)-\nu(k)-(p, q)}(z) \hat{v}_{\nu(k)}(z)\right] \\
& =\left(\frac{z+1}{2}\right)^{2}\left[\sum_{-K \leq p, q \leq K} a_{p, q} \sum_{k=1}^{\left|\mathcal{B}_{\text {Int }}\right|}\left(\hat{f}_{(j, \ell)-\nu(k)-(p, q)}(z) \hat{v}_{\nu(k)}(z)\right)\right] \\
& =\left(\frac{z+1}{2}\right)^{2} \sum_{-K \leq p, q \leq K} a_{p, q} \hat{u}_{(j, \ell)-(p, q)}(z),
\end{aligned}
$$

which leads to (12) after inversion by the $\mathcal{Z}$-transform. Finally, we obtain

$$
u_{j, \ell}^{0}=\sum_{k=1}^{\left|\mathcal{B}_{\text {Int }}\right|} f_{(j, \ell)-\nu(k)}^{0} v_{\nu(k)}^{0}=0
$$

and

$$
u_{j, \ell}^{1}=\sum_{k=1}^{\left|\mathcal{B}_{\text {Int }}\right|}\left(f_{(j, \ell)-\nu(k)}^{0} v_{\nu(k)}^{1}+f_{(j, \ell)-\nu(k)}^{1} v_{\nu(k)}^{0}\right)=0
$$

from (13).

From Lemma 2, we deduce that the function $u_{j, \ell}^{n}$, defined by $(11)$, for $(j, \ell) \in \mathbb{Z}^{2} / \mathcal{R}_{J}(0,0)$, satisfies the governing fully discretized peridynamics on the exterior domain. Thus, an expression of the $\mathrm{ABC}$ given by (11) can be obtained at the boundary if one can compute $\left\{v_{\nu(k)}^{n}\right\}_{n}$, for $1 \leq$ $k \leq\left|\mathcal{B}_{\text {Int }}\right|$. This question is considered in the following Lemma.

Lemma 3. Let us assume that $\tau^{2} \leq\left|a_{0,0}\right|^{-1} / 4$, with $a_{0,0}$ given by (4). For $\left|\mathcal{B}_{\text {Int }}\right|$ given sequences $\left\{u_{\nu(j)}^{n}\right\}_{n}$, such that $u_{\nu(j)}^{0}=0, u_{\nu(j)}^{1}=0$ and $u_{\nu(j)}^{n}=0$, for $n \geq N+1$, one can find $\left|\mathcal{B}_{\text {Int }}\right|$ sequences $\left\{v_{\nu(k)}^{n}\right\}_{n}$, with $1 \leq k \leq\left|\mathcal{B}_{\text {Int }}\right|$, satisfying

$$
u_{\nu(j)}^{n}=\sum_{k=1}^{\left|\mathcal{B}_{\text {Int }}\right|}\left(f_{\nu(j)-\nu(k)} \star v_{\nu(k)}\right)^{n}, \quad 1 \leq j \leq\left|\mathcal{B}_{\text {Int }}\right| .
$$

Furthermore, we have

$$
\sum_{(j, \ell) \in \mathbb{Z}^{2} / \mathcal{R}_{J}(0,0)}\left|u_{j, \ell}^{n}\right|^{2}<+\infty
$$

where

$$
u_{j, \ell}^{n}=\sum_{k=1}^{\left|\mathcal{B}_{\text {Int }}\right|}\left(f_{(j, \ell)-\nu(k)} \star v_{\nu(k)}\right)^{n}
$$

for $(j, \ell) \in \mathbb{Z}^{2} / \mathcal{R}_{J}(0,0)$. 
Proof of Lemma 3. Let us note that $u_{\nu(j)}^{n}=0$, for $n \geq N+1$ and $1 \leq j \leq\left|\mathcal{B}_{\text {Int }}\right|$. Thus, $\widehat{u}_{\nu(j)}(z)$ is analytic for $z \neq 0$ and $1 \leq j \leq\left|\mathcal{B}_{\text {Int }}\right|$. To get (14), we only need to solve an equivalent version, namely,

$$
\widehat{u}_{\nu(j)}=\sum_{k=1}^{\left|\mathcal{B}_{\text {Int }}\right|} \widehat{f}_{\nu(j)-\nu(k)}(z) \widehat{v}_{\nu(k)}(z), \quad 1 \leq j \leq\left|\mathcal{B}_{\text {Int }}\right| .
$$

Since $\widehat{f}_{\nu(j)-\nu(k)}(z)=\frac{\widehat{g}_{\nu(j)-\nu(k)}(z)}{\widehat{g}_{0,0}(z)},(16)$ can be written as

$$
\widehat{u}_{\nu(j)}=\sum_{k=1}^{\left|\mathcal{B}_{\text {Int }}\right|} \widehat{g}_{\nu(j)-\nu(k)}(z) \widehat{v}_{\nu(k)} \widehat{g}_{0,0}^{-1}, \quad 1 \leq j \leq\left|\mathcal{B}_{\text {Int }}\right| .
$$

Recalling now that $\mathbf{I}[\widehat{u}]=\left(\widehat{u}_{\nu(1)}, \cdots, \widehat{u}_{\nu\left(\left|\mathcal{B}_{\text {Int }}\right|\right)}\right)^{T}$ and $\mathbf{I}[\widehat{v}]=\left(\widehat{v}_{\nu(1)}, \cdots, \widehat{v}_{\nu\left(\left|\mathcal{B}_{\text {Int }}\right|\right)}\right)^{T}$, then (17) can be rewritten in the form of $\mathbf{I}[\widehat{u}]=\widehat{\mathbb{A}} \mathbf{I}[\widehat{v}]$, where $\widehat{\mathbb{A}}$ is the $\left|\mathcal{B}_{\text {Int }}\right| \times\left|\mathcal{B}_{\text {Int }}\right|$ matrix such that $\widehat{\mathbb{A}}_{j, k}(z)=$ $\widehat{g}_{|\nu(j)-\nu(k)|}(z) \widehat{g}_{0,0}^{-1}$ because $\widehat{g}_{\nu(j)-\nu(k)}=\widehat{g}_{\nu(k)-\nu(j)}$. In addition, for large values of $|z|$, we have

$$
\begin{aligned}
& \widehat{g}_{j, \ell}(z)=\frac{1}{4 \pi^{2}} \int_{0}^{2 \pi} \int_{0}^{2 \pi} \rho_{\text {Int }}^{-1}(z, x, y) e^{-\mathrm{i} j x-\mathrm{i} \ell y} d x d y \\
& =\sum_{m=0}^{\infty} \frac{1}{4 \pi^{2}} \frac{\tau^{2}}{z^{m+2}} \int_{0}^{2 \pi} \int_{0}^{2 \pi} \frac{\left(2-\frac{1}{z}+\frac{\tau^{2}}{4}\left(2+\frac{1}{z}\right) A_{\text {Int }}(x, y)\right)^{m}}{\left(1-\tau^{2} A_{\text {Int }}(x, y) / 4\right)^{m+1}} e^{-\mathrm{i} j x-\mathrm{i} \ell y} d x d y \\
& =\frac{\tau^{2}}{4 \pi^{2} z^{2}} \int_{0}^{2 \pi} \int_{0}^{2 \pi} \frac{1}{\left(1-\tau^{2} A_{\text {Int }}(x, y) / 4\right)} e^{-\mathrm{i} j x-\mathrm{i} \ell y} d x d y+\mathcal{O}\left(\frac{\tau^{2}}{z^{3}}\right)=\frac{\gamma_{j, \ell}}{z^{2}}+\mathcal{O}\left(\frac{\tau^{2}}{z^{3}}\right),
\end{aligned}
$$

where

$$
\gamma_{j, \ell}=\frac{\tau^{2}}{4 \pi^{2}} \int_{0}^{2 \pi} \int_{0}^{2 \pi} \sum_{m=0}^{\infty}\left(\frac{\tau^{2} A_{\text {Int }}(x, y)}{4}\right)^{m} e^{-\mathrm{i} j x-\mathrm{i} \ell y} d x d y=\tau^{2} \delta_{j, \ell}^{0,0}+\mathcal{O}\left(\tau^{3}\right) .
$$

Thus, we derive that: $\widehat{\mathbb{A}}=\mathbb{I}+\mathcal{O}(\tau)+\mathcal{O}\left(z^{-1}\right)$, where $\mathbb{I}$ is the $\left|\mathcal{B}_{\text {Int }}\right| \times\left|\mathcal{B}_{\text {Int }}\right|$ identity matrix. Therefore, for large values of $|z|$ and small values of $|\tau|$, we have: $\mathbf{I}[\widehat{u}] \approx \mathbb{I} \mathbf{I}[\widehat{v}]$, and conclude that (17) has a unique sequence solution $\mathbf{I}[\widehat{v}]$, which leads to (16).

In addition, we have

$$
\begin{aligned}
\widehat{g}_{j, \ell}(z)= & \frac{1}{4 \pi^{2}} \int_{0}^{2 \pi} \int_{0}^{2 \pi} \rho_{\text {Int }}^{-1}(z, x, y) e^{-\mathrm{i} j x-\mathrm{i} \ell y} d x d y=\frac{1}{-\mathrm{i} j} \frac{1}{4 \pi^{2}} \int_{0}^{2 \pi} \int_{0}^{2 \pi} \rho_{\text {Int }}^{-1}(z, x, y) e^{-\mathrm{i} \ell y} d\left(e^{-\mathrm{i} j x}\right) d y \\
= & \frac{1}{\mathrm{i} j} \frac{1}{4 \pi^{2}} \int_{0}^{2 \pi} \int_{0}^{2 \pi} \frac{\partial\left(\rho_{\text {Int }}^{-1}\right)}{\partial x} e^{-\mathrm{i} \ell y} e^{-\mathrm{i} j x} d x d y=-\frac{1}{j \ell} \frac{1}{4 \pi^{2}} \int_{0}^{2 \pi} \int_{0}^{2 \pi} \frac{\partial^{2}\left(\rho_{\text {Int }}^{-1}\right)}{\partial x \partial y} e^{-\mathrm{i} \ell y} e^{-\mathrm{i} j x} d x d y .
\end{aligned}
$$

Let us assume that $\tau^{2} \leq\left|a_{0,0}\right|^{-1} / 4$. Then, from $|z|>4$, we have

$$
\left|\frac{(z-1)^{2}}{\tau^{2}}-\frac{(z+1)^{2}}{4} A_{\text {Int }}\right| \geq \frac{(|z|-1)^{2}}{\tau^{2}}-\frac{(|z|+1)^{2}}{4} 2\left|a_{0,0}\right|>\frac{9}{\tau^{2}}-\frac{25}{2}\left|a_{0,0}\right|>\frac{47}{2}\left|a_{0,0}\right| .
$$

By (9), it is clear that $\widehat{g}_{j, \ell}(z)$ is analytic for $|z| \geq 4$ by (18). Therefore, for $|z| \geq 4$, one has

$$
\left|\widehat{g}_{j, \ell}(z)\right|=\left|\frac{1}{j \ell} \frac{1}{4 \pi^{2}}\left(\int_{0}^{2 \pi} \int_{0}^{2 \pi} \frac{\partial^{2}\left(\rho_{\text {Int }}^{-1}\right)}{\partial x \partial y} e^{-\mathrm{i} \ell y} e^{-\mathrm{i} j x} d x d y\right)\right| \leq \frac{1}{j \ell} \max _{(x, y) \in[0,2 \pi]^{2},|z| \geq 4}\left|\frac{\partial^{2}\left(\rho_{\text {Int }}^{-1}\right)}{\partial x \partial y}\right| \leq \frac{C}{j \ell} .
$$


Similarly, we have: $\left|\widehat{g}_{0, \ell}(z)\right| \leq C / \ell$. As a consequence, for the computational domain $\mathcal{R}_{J}(0,0)$ with fixed size $2 J+1$ (as stated in Section 2.2 ), with $(j, \ell) \in \mathbb{Z}^{2} / \mathcal{R}_{J}(0,0)$ and $|z| \geq 4$, one gets

$$
\left|\widehat{u}_{j, \ell}\right|=\left|\sum_{k=1}^{\left|\mathcal{B}_{\text {Int }}\right|} \widehat{g}_{(j, \ell)-\nu(k)} \widehat{v}_{\nu(k)}\left(\widehat{g}_{0,0}\right)^{-1}\right| \leq \frac{C\left|\mathcal{B}_{\text {Int }}\right|}{|j \pm J|}, \quad \text { for }|j|>J .
$$

We also have: $\left|\widehat{u}_{j, \ell}\right| \leq C\left|\mathcal{B}_{\text {Int }}\right||\ell \pm J|^{-1}$, for $|\ell|>J$, and $\left|\widehat{u}_{j, \ell}\right| \leq \frac{C\left|\mathcal{B}_{\text {Int }}\right|}{|j \pm J||\ell \pm J|}$ for $|\ell|>J$ and $|j|>J$. For $N$ fixed and $n \leq N$, the above two inequalities imply that

$$
\begin{aligned}
& \left|u_{j, \ell}^{n}\right|=\left|\frac{1}{2 \pi \mathrm{i}} \int_{|z|=4} \widehat{u}_{j, \ell}(z) z^{n-1} d z\right| \leq \frac{C\left|\mathcal{B}_{\text {Int }}\right|}{|| j|-J|} \int_{|z|=4}|z|^{n-1}|d z| \leq \frac{C\left|\mathcal{B}_{\text {Int }}\right| 4^{N}}{|| j|-J|}, \quad \text { for }|j|>J \\
& \left|u_{j, \ell}^{n}\right| \leq \frac{C\left|\mathcal{B}_{\text {Int }}\right| 4^{N}}{|| \ell|-J|}, \quad \text { for }|\ell|>J \\
& \left|u_{j, \ell}^{n}\right| \leq \frac{C\left|\mathcal{B}_{\text {Int }}\right| 4^{N}}{|| \ell|-J||| j|-J|}, \quad \text { for }|\ell|>J,|j|>J
\end{aligned}
$$

By using (19), we obtain

$$
\begin{aligned}
& \sum_{(j, \ell) \in \mathbb{Z}^{2} / \mathcal{R}_{J}(0,0)}\left|u_{j, \ell}^{n}\right|^{2}=\sum_{|\ell|>J,|j| \leq J}\left|u_{j, \ell}^{n}\right|^{2}+\sum_{|\ell| \leq J,|j|>J}\left|u_{j, \ell}^{n}\right|^{2}+\sum_{|\ell|>J,|j|>J}\left|u_{j, \ell}^{n}\right|^{2} \\
& \leq C\left|\mathcal{B}_{\text {Int }}\right|^{2}(16)^{N} \sum_{|j| \leq J} \sum_{\ell=J+1}^{\infty} \frac{1}{(\ell-J)^{2}}+C\left|\mathcal{B}_{\text {Int }}\right|^{2}(16)^{N} \sum_{|\ell| \leq J} \sum_{j=J+1}^{\infty} \frac{1}{(j-J)^{2}} \\
& +C\left|\mathcal{B}_{\text {Int }}\right|^{2}(16)^{N} \sum_{j=J+1}^{\infty} \sum_{\ell=J+1}^{\infty} \frac{1}{(j-J)^{2}(\ell-J)^{2}}<+\infty .
\end{aligned}
$$

Based on Lemmas 2 and 3, we can now prove Theorem 1 .

Proof of Theorem 1. From Lemma 3, there exist $\left|\mathcal{B}_{\text {Int }}\right|$ sequences $\left\{v_{\nu(k)}^{n}\right\}_{n}$, with $1 \leq k \leq\left|\mathcal{B}_{\text {Int }}\right|$, such that (14) holds for the $\left|\mathcal{B}_{\text {Int }}\right|$ interior layer values $\left\{u_{\nu(j)}^{n}\right\}_{n}$, with $1 \leq j \leq\left|\mathcal{B}_{\text {Int }}\right|$. Now, $\left\{u_{j, \ell}^{n}\right\}_{n}$ can be defined from the sequences $\left\{v_{\nu(k)}^{n}\right\}_{n}$ by $(11)$, with $(j, \ell) \in \mathbb{Z}^{2} / \mathcal{R}_{J}(0,0)$. Therefore, the sequences $\left\{u_{\mu(j)}^{n}\right\}_{n}$ satisfy (12), for $1 \leq j \leq\left|\mathcal{B}_{\text {Ext }}\right|$, by Lemma 2. Thus, we prove that (11) and (14) provide the boundary condition, namely

$$
\left\{\begin{array}{l}
u_{\mu(j)}^{n}=\sum_{k=1}^{\left|\mathcal{B}_{\text {Int }}\right|}\left(f_{|\mu(j)-\nu(k)|} \star v_{\nu(k)}\right)^{n}, \quad 1 \leq j \leq\left|\mathcal{B}_{\text {Ext }}\right|, \\
u_{\nu(\ell)}^{n}=\sum_{k=1}^{K}\left(f_{|\nu(\ell)-\nu(k)|} \star v_{\nu(k)}\right)^{n}, \quad 1 \leq \ell \leq\left|\mathcal{B}_{\text {Int }}\right|
\end{array}\right.
$$

since $f_{j, \ell}^{n}=f_{j,-\ell}^{n}=f_{-j, \ell}^{n}=f_{-j,-\ell}^{n}$. Now, by eliminating $\widehat{u}(z)$ after applying the $\mathcal{Z}$-transform to (20), we directly obtain

$$
u_{\mu(j)}^{n}=\sum_{k=1}^{\left|\mathcal{B}_{\text {Int }}\right|}\left(\mathbb{C}_{j, k} \star u_{\nu(k)}\right)^{n}, \quad 1 \leq j \leq\left|\mathcal{B}_{\text {Ext }}\right|,
$$

where $\widehat{\mathbb{C}}(z)=\widehat{\mathbb{B}}(z) \widehat{\mathbb{A}}^{-1}(z)$ such that $\left(\mathbb{A}^{n}\right)_{j, k}=f_{|\mu(j)-\nu(k)|}^{n}$, with $1 \leq j, k \leq\left|\mathcal{B}_{\text {Int }}\right|$, and $\left(\mathbb{B}^{n}\right)_{j, k}=$ $f_{|\nu(j)-\nu(k)|}^{n}$, with $1 \leq j \leq\left|\mathcal{B}_{\text {Ext }}\right|, 1 \leq k \leq\left|\mathcal{B}_{\text {Int }}\right|$. 
Since $f_{j, \ell}^{n}=f_{j,-\ell}^{n}=f_{-j, \ell}^{n}=f_{-j,-\ell}^{n}$, we only need to compute $f_{j, \ell}^{n}$, with $0 \leq j, \ell \leq K+2 J$. The term $\widehat{\mathbb{C}}(z)$ is determined in Subsection 2.3 by the sequences $\left\{f_{j, \ell}^{n}\right\}_{n}$ through the relation: $\widehat{\mathbb{C}}(z)=\widehat{\mathbb{B}}(z) \widehat{\mathbb{A}}^{-1}(z)$. Furthermore, since

$$
\widehat{\mathbb{B}}_{j, k}(z)=\frac{\widehat{g}_{|\mu(j)-\nu(k)|}(z)}{\widehat{g}_{0,0}(z)}, \quad \text { and } \quad \widehat{\mathbb{A}}_{j, k}(z)=\frac{\widehat{g}_{|\nu(j)-\nu(k)|}(z)}{\widehat{g}_{0,0}(z)},
$$

the matrices $\widehat{\mathbb{A}}$ and $\widehat{\mathbb{B}}$ can be modified as $\widehat{\mathbb{B}}_{j, k}(z)=\widehat{g}_{|\mu(j)-\nu(k)|}(z)$ and $\widehat{\mathbb{A}}_{j, k}(z)=\widehat{g}_{|\nu(j)-\nu(k)|}(z)$ to obtain $\widehat{\mathbb{C}}$ from $\widehat{\mathbb{C}}=\widehat{\mathbb{B}} \widehat{\mathbb{A}}^{-1}$. Hence, computing the functions $\widehat{g}_{j, \ell}(z)$ instead of $\widehat{f}_{j, \ell}(z)$ is sufficient.

\subsection{Computation of the function $\left\{g_{j, \ell}^{n}\right\}_{n}$ and Green's function $\left\{\mathbb{C}^{n}\right\}_{n}$}

To derive the $\operatorname{ABC}(7)$, we have just seen that we only need to compute the sequences $\left\{g_{j, \ell}^{n}\right\}_{n}$, for $0 \leq \ell \leq j \leq 2 J+K$, with $\left\{g_{j, \ell}^{n}\right\}_{n}$ the inverse $\mathcal{Z}$-transform of $\widehat{g}_{j, \ell}(z)$. By Lemma 1 , the function $\widehat{g}_{j, \ell}(z)$, for $(j, \ell) \in \mathbb{Z}^{2}$, satisfies

$$
\left(\frac{z-1}{\tau}\right)^{2} \widehat{g}_{j, \ell}(z)=\left(\frac{z+1}{2}\right)^{2}\left[\sum_{-K \leq k, m \leq K} a_{k, m} \widehat{g}_{j-k, \ell-m}(z)\right]+\delta_{j, \ell}^{0,0},
$$

leading to

$$
\begin{aligned}
& D_{\tau}^{-} D_{\tau}^{+} g_{j, \ell}^{n}=E^{-} E^{+} L_{\delta, h} g_{j, \ell}^{n}+\delta_{j, \ell}^{0,0}, \\
& g_{j, \ell}^{0}=0, g_{j, \ell}^{1}=0 .
\end{aligned}
$$

Let us define $\Delta_{k, m}^{x} g_{j, \ell}^{n}=g_{j+k, \ell+m}^{n}-g_{j-k, \ell+m}^{n}$. Then, the following result holds.

Lemma 4. The sequences $\left\{g_{j, \ell}^{n}\right\}_{n}$, for $(j, \ell) \in \mathbb{Z}^{2}$ and $n \geq 2$, satisfy the recursive relation

$$
\begin{aligned}
& n \sum_{k, m=1}^{K} a_{k, m}\left(\Delta_{k, m}^{x} g_{j, \ell}^{n+2}+\Delta_{k,-m}^{x} g_{j, \ell}^{n+2}\right)+(3 n-1) \sum_{k, m=1}^{K} k a_{k, m}\left(\Delta_{k, m}^{x} g_{j, \ell}^{n+1}+\Delta_{k,-m}^{x} g_{j, \ell}^{n+1}\right) \\
& +(3 n-2) \sum_{k, m=1}^{K} k a_{k, m}\left(\Delta_{k, m}^{x} g_{j, \ell}^{n}+\Delta_{k,-m}^{x} g_{j, \ell}^{n}\right)+(n-1) \sum_{k, m=1}^{K} k a_{k, m}\left(\Delta_{k, m}^{x} g_{j, \ell}^{n-1}+\Delta_{k,-m}^{x} g_{j, \ell}^{n-1}\right) \\
& +(3 n-2) \sum_{k=1}^{K} k a_{k, 0} \Delta_{k, 0}^{x} g_{j, \ell}^{n}+(n-1) \sum_{k=1}^{K} k a_{k, 0} \Delta_{k, 0}^{x} g_{j, \ell}^{n-1}+n \sum_{k=1}^{K} k a_{k, 0} \Delta_{k, 0}^{x} g_{j, \ell}^{n+2} \\
& +(3 n-1) \sum_{k=1}^{K} k a_{k, 0} \Delta_{k, 0}^{x} g_{j, \ell}^{n+1}=\frac{16 j}{\tau^{2}} g_{j, \ell}^{n}-\frac{16 j}{\tau^{2}} g_{j, \ell}^{n+1} .
\end{aligned}
$$

Proof of Lemma 4. For any $(j, \ell) \in \mathbb{Z}^{2}$, we define $\hat{h}_{j, \ell}(z)=(z+1)^{2} \hat{g}_{j, \ell}(z)$ and $\hat{h}_{j-k, \ell-m}-$ $\hat{h}_{j+k, \ell-m}=-\Delta_{k,-m}^{x} \hat{h}_{j, \ell}$. Then one gets 


$$
\begin{aligned}
& \frac{d}{d z}\left(\sum_{k, m=1}^{K} k a_{k, m}\left[-\Delta_{k,-m}^{x} \hat{h}_{j, \ell}-\Delta_{k, m}^{x} \hat{h}_{j, \ell}\right]-\sum_{k=1}^{K} k a_{k, 0} \Delta_{k, 0}^{x} \hat{h}_{j, \ell}\right) \\
& =\frac{1}{4 \pi^{2}} \frac{d}{d z}\left[\int_{0}^{2 \pi} \int_{0}^{2 \pi} \mathrm{i}(1+z)^{2} \rho_{\text {Int }}^{-1}(z, x)\left[-\frac{\partial A_{\text {Int }}(x, y)}{\partial x}\right] e^{-\mathrm{i} j x-\mathrm{i} \ell y} d x d y\right] \\
& =-\frac{4\left(z^{2}-1\right)}{\tau^{2}}\left(\frac{1}{4 \pi^{2}} \int_{0}^{2 \pi} \int_{0}^{2 \pi} \mathrm{i}\left[-\frac{\partial A_{\text {Int }}(x, y)}{\partial x}\right] \rho_{\text {Int }}^{-2}(z, x, y) e^{-\mathrm{i} j x-\mathrm{i} \ell y} d x d y\right) \\
& =\frac{16 \mathrm{i}(z-1)}{(z+1)^{3} \tau^{2}}\left(\frac{1}{4 \pi^{2}} \int_{0}^{2 \pi} \int_{0}^{2 \pi}\left[-\frac{1}{4} \frac{\partial A_{\text {Int }}(x, y)}{\partial x}\right]\left[-(1+z)^{4} \rho_{\text {Int }}^{-2}(z, x, y)\right] e^{-\mathrm{i} j x-\mathrm{i} \ell y} d x d y\right) \\
& =\frac{16 \mathrm{i}(z-1)}{(z+1)^{3} \tau^{2}}\left(\frac{1}{4 \pi^{2}} \int_{0}^{2 \pi} \int_{0}^{2 \pi}-\left((1+z)^{-2} \rho_{\text {Int }}(z, x, y)\right)^{-2} e^{-\mathrm{i} j x-\mathrm{i} \ell y} d\left((1+z)^{-2} \rho_{\text {Int }}(z, x, y)\right) d y\right) \\
& =-\frac{16 j(z-1)}{(z+1)^{3} \tau^{2}}\left(\frac{1}{4 \pi^{2}} \int_{0}^{2 \pi} \int_{0}^{2 \pi}\left((1+z)^{-2} \rho_{\text {Int }}(z, x, y)\right)^{-1} e^{-\mathrm{i} j x-\mathrm{i} \ell y} d x d y\right)=-\frac{16 j(z-1)}{(z+1) \tau^{2}} \hat{g}_{j, \ell},
\end{aligned}
$$

which leads, with $\Delta_{k, m}^{x} \hat{g}_{j, \ell}=\hat{g}_{j+k, \ell+m}-\hat{g}_{j-k, \ell+m}$, to

$$
\begin{aligned}
& -\frac{16 j(z-1)}{(z+1) \tau^{2}} \hat{g}_{j, \ell}+2(z+1) \sum_{k, m=1}^{K} k a_{k, m}\left[-\Delta_{k, m}^{x} \hat{g}_{j, \ell}-\Delta_{k,-m}^{x} \hat{g}_{j, \ell}\right]-2(z+1) \sum_{k=1}^{K} k a_{k, 0} \Delta_{k, 0}^{x} \hat{g}_{j, \ell} \\
& +(z+1)^{2} \sum_{k, m=1}^{K} k a_{k, m}\left[-\Delta_{k, m}^{x} \frac{d \hat{g}_{j, \ell}}{d z}-\Delta_{k,-m}^{x} \frac{d \hat{g}_{j-k, \ell-m}}{d z}\right]-(z+1)^{2} \sum_{k=1}^{K} k a_{k, 0} \Delta_{k, 0}^{x} \frac{d \hat{g}_{j-k, \ell}}{d z} .
\end{aligned}
$$

After some manipulations, we obtain

$$
\begin{aligned}
& 2 \sum_{k, m=1}^{K} k a_{k, m}\left(-\Delta_{k, m}^{x} g_{j, \ell}^{n}-\Delta_{k,-m}^{x} g_{j, \ell}^{n}\right)+4 \sum_{k, m=1}^{K} k a_{k, m}\left(-\Delta_{k, m}^{x} g_{j, \ell}^{n+1}-\Delta_{k,-m}^{x} g_{j, \ell}^{n+1}\right) \\
& +2 \sum_{k, m=1}^{K} k a_{k, m}\left(-\Delta_{k, m}^{x} g_{j, \ell}^{n+2}-\Delta_{k,-m}^{x} g_{j, \ell}^{n+2}\right)-2 \sum_{k=1}^{K} k a_{k, 0} \Delta_{k, 0}^{x} g_{j, \ell}^{n}-4 \sum_{k=1}^{K} k a_{k, 0} \Delta_{k, 0}^{x} g_{j, \ell}^{n+1} \\
& -2 \sum_{k=1}^{K} k a_{k, 0} \Delta_{k, 0}^{x} g_{j, \ell}^{n+2}+\sum_{k, m=1}^{K} k a_{k, m}(n-1)\left(\Delta_{k, m}^{x} g_{j, \ell}^{n-1}+\Delta_{k,-m}^{x} g_{j, \ell}^{n-1}\right) \\
& +3 \sum_{k, m=1}^{K} k a_{k, m} n\left(\Delta_{k, m}^{x} g_{j, \ell}^{n}+\Delta_{k,-m}^{x} g_{j, \ell}^{n}\right)+3 \sum_{k, m=1}^{K} k a_{k, m}(n+1)\left(\Delta_{k, m}^{x} g_{j, \ell}^{n+1}+\Delta_{k,-m}^{x} g_{j, \ell}^{n+1}\right) \\
& +\sum_{k, m=1}^{K} k a_{k, m}(n+2)\left(\Delta_{k, m}^{x} g_{j, \ell}^{n+2}+\Delta_{k,-m}^{x} g_{j, \ell}^{n+2}\right)+\sum_{k=1}^{K} k a_{k, 0}(n-1) \Delta_{k, 0}^{x} g_{j, \ell}^{n-1}+3 \sum_{k=1}^{K} k a_{k, 0} n \Delta_{k, 0}^{x} g_{j, \ell}^{n} \\
& +3 \sum_{k=1}^{K} k a_{k, 0}(n+1) \Delta_{k, 0}^{x} g_{j, \ell}^{n+1}+\sum_{k=1}^{K} k a_{k, 0}(n+2) \Delta_{k, 0}^{x} g_{j, \ell}^{n+2}=\frac{16 j}{\tau^{2}} g_{j, \ell}^{n}-\frac{16 j}{\tau^{2}} g_{j, \ell}^{n+1} .
\end{aligned}
$$

With some additional computations and simplifications, we prove (23).

We denote by $r_{j, \ell}$ the relation (23) for the indices $(j, \ell)$, and we set $L=2 J+K$. We now present the numerical algorithm to compute $g_{j, \ell}$, with $(i, j) \in \Omega_{1}=\{(j, \ell): 0 \leq \ell \leq j \leq L\}$. Since the indices have to be carefully checked, we report on Figure 2 the particular case $J=3, K=2$ and 


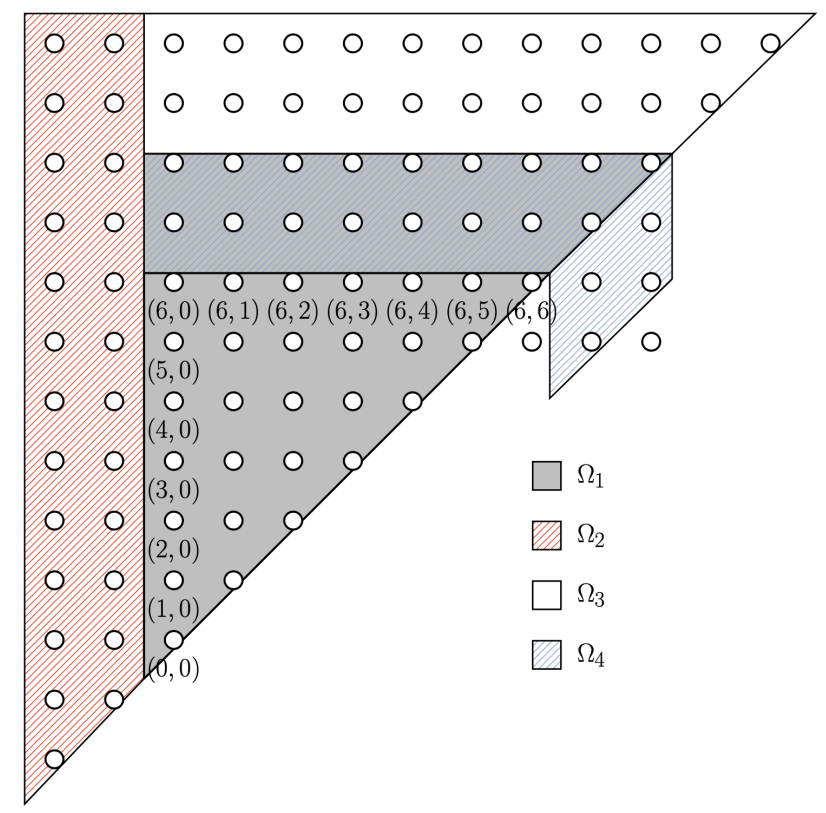

Figure 2: Indices for closing the discrete peridynamics system $(21)(J=3, K=2$ and $L=8)$.

$L=8$ as an example for the reader. We use the $(1+L)(2+L) / 2$ relations $(21)$ with $(i, j) \in \Omega_{1}$ (the light gray domain in Figure 2) to compute these sequences $\left\{g_{j, \ell}^{n}\right\}_{n}$ with $(i, j) \in \Omega_{1}$. To this end, based on $g_{\ell, j}^{n}=g_{j, \ell}^{n}$, the sequences $\left\{g_{j, \ell}^{n}\right\}_{n}$, for $(j, \ell) \in \Omega_{2}=\{(j, \ell): \ell \leq j,-K \leq j \leq L+K,-K \leq \ell \leq-1\}$ (the red hatched domain in Figure 2) and $(j, \ell) \in \Omega_{3}=\{(j, \ell): \ell \leq j, L+1 \leq j \leq L+K, 0 \leq$ $\ell \leq L+K\}$ (the white domain in Figure 2), are needed to close (21). Furthermore, the $g_{j, \ell}^{n}$, for $\ell<0$, can be obtained by $g_{j, \ell}^{n}=g_{j,-\ell}^{n}$, and $g_{j, \ell}^{n}$, for $j<\ell$, are deduced from $g_{j, \ell}^{n}=g_{\ell, j}^{n}$. Thus, the $\left\{g_{j, \ell}^{n}\right\}_{n}$ for $(j, \ell) \in \Omega_{2}$, can be obtained from $\left\{g_{j, \ell}^{n}\right\}_{n}$, for $(j, \ell) \in \Omega_{1} \cup \Omega_{3}$. Therefore, we only need to find $K(L+1)+(K+1) K / 2$ additional relations to close the $(1+L)(2+L) / 2$ relations (21), for $(i, j) \in \Omega_{1}$, in order to solve $\left\{g_{j, \ell}^{n}\right\}_{n}$, for $(i, j) \in \Omega_{1} \cup \Omega_{3}$. To this end, the $K(L+1)+(K+1) K / 2$ relations $r_{j, \ell}$, for $(j, \ell) \in \Omega_{4}=\{(j, \ell): L-K+1 \leq j \leq L, 0 \leq \ell \leq L\} \cup\{(j, \ell): \ell \leq j, L-2 K+1 \leq$ $j \leq L-K, L-K+1 \leq \ell \leq L\}$ (the blue hatched domain in Figure 2), will be chosen to close the $(1+L)(2+L) / 2$ relations $(21)$.

Now, we determine the discrete system for $\left\{g_{j, \ell}^{n}\right\}_{n}$ for $(i, j) \in \Omega_{1}$. After getting the value of $\left\{g_{j, \ell}^{n}\right\}_{n}$ for $(j, \ell) \in \Omega_{1}$, we obtain $\mathbb{C}^{n}$ by $\mathbb{B}^{n}=(\mathbb{C} \star \mathbb{A})^{n}=\sum_{k=0}^{n} \mathbb{C}^{k} \cdot \mathbb{A}^{n-k}=\mathbb{C}^{n} \mathbb{A}^{0}+\sum_{k=0}^{n-1} \mathbb{C}^{k} \mathbb{A}^{n-k}$, or equivalently

$$
\mathbb{C}^{n}=\left(\mathbb{B}^{n}-\sum_{k=0}^{n-1} \mathbb{C}^{k} \mathbb{A}^{n-k}\right)\left(\mathbb{A}^{0}\right)^{-1}
$$

The previous numerical algorithm is applied for solving $g_{j, \ell}^{n}$, for $n \neq 2$. For the specific case $n=2$, we still need to compute $g_{j, \ell}^{2}$, for $0 \leq \ell \leq j \leq L+K$. To this end, $g_{j, \ell}^{2}$ is rewritten as

$$
g_{j, \ell}^{2}=\frac{\tau^{2}}{4 \pi^{2}} \int_{0}^{2 \pi} \int_{0}^{2 \pi} \frac{1}{\left(1-\tau^{2} A_{\operatorname{Int}}(x, y) / 4\right)} e^{-\mathrm{i} j x-\mathrm{i} \ell y} d x d y,
$$

and can be evaluated by a direct numerical quadrature. 
Remark 1. For the three-dimensional case, the previous derivations can be extended by considering

$$
\hat{g}_{j, \ell, p}(z)=\frac{1}{8 \pi^{3}} \int_{0}^{2 \pi} \int_{0}^{2 \pi} \int_{0}^{2 \pi}\left(\rho_{\text {Int }}\left(z, x_{1}, y_{1}, z_{1}\right)\right)^{-1} e^{-\mathrm{i} j x_{1}-\mathrm{i} \ell y_{1}-\mathrm{i} p z_{1}} d x_{1} d y_{1} d z_{1},
$$

with

$$
\rho_{\mathrm{Int}}\left(z, x_{1}, y_{1}, z_{1}\right):=\left(\frac{z-1}{\tau}\right)^{2}-\left(\frac{z+1}{2}\right)^{2} A_{\mathrm{Int}}\left(x_{1}, y_{1}, z_{1}\right)
$$

where

$$
A_{\text {Int }}\left(x_{1}, y_{1}, z_{1}\right):=\sum_{k, m, q=-K}^{K} a_{k, m, p} e^{-\mathrm{i} k x_{1}} e^{-\mathrm{i} m y_{1}} e^{-\mathrm{i} q z_{1}} .
$$

Then, it can be proven that

$$
D_{\tau}^{-} D_{\tau}^{+} g_{j, \ell, p}^{n}=\sum_{k, m, q=-K}^{K} a_{k, m, q} E^{-} E^{+} g_{j+k, \ell+m, p+q}^{n}+\delta_{j, \ell, p}^{0,0,0}, \quad \text { for } j, \ell, p \in \mathbb{Z},
$$

and the ABCs for the three-dimensional situation can be developed as in Subsection 2.2.

\subsection{Numerical algorithm for the fully discretized peridynamics system with $A B C$}

We give now the corresponding numerical algorithm for the reformulated fully discretized peridynamics on a bounded computational domain $\mathcal{R}_{J}(0,0)$ with the accurate absorbing boundary condition. Following Subsection 2.2, we adopt the Crank-Nicolson scheme (5) for the time integration in (1). Then, relation (7) is introduced to serve as ABC. To compute $u_{j, \ell}^{1}$, we use the Runge-Kutta 4 (RK4) scheme applied to the equivalent form of (1): $\dot{u}_{t}=v, \dot{v}_{t}=\mathcal{L}_{\delta} u$, leading to

$$
\begin{aligned}
& u_{j, \ell}^{1}=u_{j, \ell}^{0}+\left(F_{j, \ell}^{1}+2 F_{j, \ell}^{2}+2 F_{j, \ell}^{3}+F_{j, \ell}^{4}\right) \tau / 6, \quad v_{j, \ell}^{1}=v_{j, \ell}^{0}+\left(G_{j, \ell}^{1}+2 G_{j, \ell}^{2}+2 G_{j, \ell}^{3}+G_{j, \ell}^{4}\right) \tau / 6, \\
& F_{j, \ell}^{1}=v_{j, \ell}^{0}, \quad G_{j, \ell}^{1}=L_{\delta, h} u_{j, \ell}^{0}, \\
& F_{j, \ell}^{2}=v_{j, \ell}^{0}+G_{j, \ell}^{1} h / 2, \quad G_{j, \ell}^{2}=L_{\delta, h}\left(u_{j, \ell}^{0}+F_{j, \ell}^{1} h / 2\right), \\
& F_{j, \ell}^{3}=v_{j, \ell}^{0}+G_{j, \ell}^{2} h / 2, \quad G_{j, \ell}^{3}=L_{\delta, h}\left(u_{j, \ell}^{0}+F_{j, \ell}^{2} h / 2\right), \\
& F_{j, \ell}^{4}=v_{j, \ell}^{0}+G_{j, \ell}^{3} h, \quad G_{j, \ell}^{4}=L_{\delta, h}\left(u_{j, \ell}^{0}+F_{j, \ell}^{3} h\right),
\end{aligned}
$$

by setting $u_{j, \ell}^{0}=\phi^{0}\left(x_{j}, y_{\ell}\right)$ and $v_{j, \ell}^{0}=\phi^{1}\left(x_{j}, y_{\ell}\right)$. Then, one can solve $u_{j, \ell}^{n}$, for $n \geq 2$ and $(j, \ell) \in$ $\mathcal{R}_{J}(0,0)$, by (5) and (7). Since (5) is a three layers scheme, we need the values of $u_{j, \ell}^{1}$ to compute $u_{j, \ell}^{n}$, for $n \geq 2$. Thus we can use (24) to compute $u_{j, \ell}^{1}$ from $u_{j, \ell}^{0}$ in a large enough domain since the initial data are compactly supported.

\section{Properties of the kernel $\left\{\mathbb{C}^{n}\right\}_{n}$ and stability analysis}

In this section, we start by proving some properties of the kernel $\left\{\mathbb{C}^{n}\right\}_{n}$ that are next used to state the stability of the CNAC with ABC.

\subsection{Properties of the kernel $\left\{\mathbb{C}^{n}\right\}_{n}$}

From now on, we use $\bar{z}$ to denote the complex conjugate of $z \in \mathbb{C}$. We start with the following Proposition. 
Proposition 1. For sequences $\left\{u_{\nu(k)}^{n}\right\}_{n}$ located at $\nu(k)$ with, $1 \leq k \leq\left|\mathcal{B}_{\text {Int }}\right|$, such that $u_{\nu(k)}^{0}=0$ and $u_{\nu(k)}^{1}=0$, and for any positive integer $N$, the following inequality holds

$$
\begin{aligned}
& \sum_{n=1}^{N-1}\left(E^{+}(\mathbb{C} \star \mathbf{I}[u])^{n}-E^{-}(\mathbb{C} \star \mathbf{I}[u])^{n}\right)^{T} \mathbb{S}\left(E^{+}(\mathbf{I}[u])^{n}+E^{-}(\mathbf{I}[u])^{n}\right) \\
& \geq \sum_{(j, \ell) \in \mathcal{B}_{\text {Int }}} \sum_{(k, m) \in \mathcal{B}_{\text {Ext }}} a_{k-j, m-\ell}\left|E^{+} u_{k, m}^{N-1}\right|^{2} .
\end{aligned}
$$

Here, $\mathbb{S}$ is defined by $\mathbb{S}_{i, j}=a_{\mu(i)-\nu(j)}$, for $1 \leq i \leq\left|\mathcal{B}_{\text {Ext }}\right|$ and $1 \leq j \leq\left|\mathcal{B}_{\text {Int }}\right|$. The coefficients $a_{k, m}$ are defined by (4), for $|k|,|m| \leq K$.

Proof of Proposition 1. By Lemma 3, for any vector sequence $\left\{u_{\nu(k)}^{n}\right\}_{n}$ located at $\nu(k)$ such that $u_{\nu(k)}^{0}=0$ and $u_{\nu(k)}^{1}=0$, with $1 \leq k \leq\left|\mathcal{B}_{\text {Int }}\right|$, there exist some sequences $\left\{v_{\nu(k)}^{n}\right\}_{n}$ located at $\nu(k)$, with $1 \leq k \leq\left|\mathcal{B}_{\text {Int }}\right|$ and $n \leq N-1$, such that the following equality holds

$$
u_{\nu(j)}^{n}=\sum_{k=0}^{K-1}\left(f_{\nu(j)-\nu(k)} \star v_{\nu(k)}\right)^{n}, \quad 1 \leq j \leq\left|\mathcal{B}_{\text {Int }}\right| .
$$

Thus, we can define

$$
u_{j, \ell}^{n}=\sum_{k=1}^{\mathcal{B}_{\text {Int }}}\left(f_{(j, \ell)-\nu(k)} \star v_{\nu(k)}\right)^{n}, \quad(j, \ell) \in \mathbb{Z}^{2} / \mathcal{R}_{J}(0,0) .
$$

Let us remark that: $D_{\tau}^{+} D_{\tau}^{-}=\left(D_{\tau}^{+}-D_{\tau}^{-}\right) / \tau,\left(E^{+}-E^{-}\right) / \tau=\left(D_{\tau}^{+}+D_{\tau}^{-}\right) / 2$ and $E^{+} E^{-}=\left(E^{+}+\right.$ $\left.E^{-}\right) / 2$. Then, $\left\{u_{j, \ell}^{n}\right\}_{n}$, for $(j, \ell) \in \mathbb{Z}^{2} / \mathcal{R}_{J}(0,0)$, satisfy the fully discrete peridynamics system (12)

$$
\begin{aligned}
& \left(D_{\tau}^{+}-D_{\tau}^{-}\right) u_{j, \ell}^{n} / \tau=D_{\tau}^{+} D_{\tau}^{-} u_{j, \ell}^{n} \\
& =\sum_{(k, m) \in \mathcal{R}_{K}(0,0)} a_{k, m} E^{+} E^{-} u_{j+k, \ell+m}^{n}=\sum_{(k, m) \in \mathcal{R}_{K}(0,0)} a_{k, m}\left(E^{+}+E^{-}\right) u_{j+k, \ell+m}^{n} / 2 .
\end{aligned}
$$

Multiplying the above equality by $\left(D_{\tau}^{+}+D_{\tau}^{-}\right) u_{j, \ell}^{n}$, and summing up all the terms for $(j, \ell) \in$ $\mathbb{Z}^{2} / \mathcal{R}_{J}(0,0)$, we get

$$
\begin{aligned}
& \sum_{\mathbb{Z}^{2} / \mathcal{R}_{J}(0,0)}\left(\left(D_{\tau}^{+}-D_{\tau}^{-}\right) u_{j, \ell}^{n}\right)\left(D_{\tau}^{+}+D_{\tau}^{-}\right) u_{j, \ell}^{n} / \tau \\
= & \sum_{\mathbb{Z}^{2} / \mathcal{R}_{J}(0,0)} \sum_{(k, m) \in \mathcal{R}_{K}(0,0)} a_{k, m}\left(\left(E^{+}+E^{-}\right) u_{j+k, \ell+m}^{n}\right)\left(D_{\tau}^{+}+D_{\tau}^{-}\right) u_{j, \ell}^{n} / 2 \\
= & \sum_{\mathbb{Z}^{2} / \mathcal{R}_{J}(0,0)} \sum_{(k, m) \in \mathcal{R}_{K}(0,0)} a_{k, m}\left(\left(E^{+}+E^{-}\right) u_{j+k, \ell+m}^{n}\right)\left(E^{+}-E^{-}\right) u_{j, \ell}^{n} /(2 \tau) .
\end{aligned}
$$


By (15), the left hand side of (26) satisfies

$$
\begin{aligned}
& \left|\sum_{\mathbb{Z}^{2} / \mathcal{R}_{J}(0,0)} \sum_{(k, m) \in \mathcal{R}_{K}(0,0)} \frac{a_{k, m}}{2 \tau}\left(\left(E^{+}+E^{-}\right) u_{j+k, \ell+m}^{n}\right)\left(E^{+}-E^{-}\right) u_{j, \ell}^{n}\right| \\
& \leq \frac{C}{\tau} \sum_{\mathbb{Z}^{2} / \mathcal{R}_{J}(0,0)} \sum_{(k, m) \in \mathcal{R}_{K}(0,0)}\left|a_{k, m}\right|\left(\left|u_{j+k, \ell+m}^{n+1}\right|^{2}+\left|u_{j+k, \ell+m}^{n+1}\right|^{2}+\left|u_{j+k, \ell+m}^{n}\right|^{2}+\left|u_{j+k, \ell+m}^{n}\right|^{2}\right. \\
& \left.+\left|u_{j+k, \ell+m}^{n-1}\right|^{2}+\left|u_{j+k, \ell+m}^{n-1}\right|^{2}\right) \\
& +\frac{C}{\tau} \sum_{\mathbb{Z}^{2} / \mathcal{R}_{J}(0,0)} \sum_{(k, m) \in \mathcal{R}_{K}(0,0)}\left|a_{k, m}\right|\left(\left|u_{j, \ell}^{n+1}\right|^{2}+\left|u_{j, \ell}^{n+1}\right|^{2}+\left|u_{j, \ell}^{n}\right|^{2}+\left|u_{j, \ell}^{n}\right|^{2}+\left|u_{j, \ell}^{n-1}\right|^{2}+\left|u_{j, \ell}^{n-1}\right|^{2}\right) \\
& \leq \frac{C\left|a_{0,0}\right|}{\tau}(2 K+1)^{2} \sum_{(j, \ell) \in\left\{\mathbb{Z}^{2} / \mathcal{R}_{J-K}(0,0)\right\}}\left(\left.\left|u_{j, \ell}^{n+1}\right|^{2}|+| u_{j, \ell}^{n}\right|^{2}+\left|u_{j, \ell}^{n-1}\right|^{2}\right)<+\infty,
\end{aligned}
$$

which indicates that the series in (26) is absolutely convergent. Thus, we can exchange the summation order of the series expansion (26)

$$
\begin{aligned}
& 2 \sum_{\mathbb{Z}^{2} / \mathcal{R}_{J}(0,0)} \frac{\left|\left(D_{\tau}^{+}\right) u_{j, \ell}^{n}\right|^{2}-\left|\left(D_{\tau}^{-}\right) u_{j, \ell}^{n}\right|^{2}}{\tau} \\
= & \sum_{(k, m) \in \mathcal{R}_{K}(0,0)} a_{k, m} \sum_{\mathbb{Z}^{2} / \mathcal{R}_{J}(0,0)}\left(\left(E^{+}+E^{-}\right) u_{j+k, \ell+m}^{n}\right)\left(E^{+}-E^{-}\right) u_{j, \ell}^{n} / \tau \\
= & \sum_{(k, m) \in \mathcal{R}_{K}(0,0)} a_{k, m} \sum_{\mathbb{Z}^{2} / \mathcal{R}_{J}(0,0)}\left(E^{+} u_{j+k, \ell+m}^{n} E^{+} u_{j, \ell}^{n}-E^{-} u_{j+k, \ell+m}^{n} E^{-} u_{j, \ell}^{n}\right) / \tau \\
+ & \sum_{(k, m) \in \mathcal{R}_{K}(0,0)} a_{k, m} \sum_{\mathbb{Z}^{2} / \mathcal{R}_{J}(0,0)}\left(E^{-} u_{j+k, \ell+m}^{n} E^{+} u_{j, \ell}^{n}-E^{+} u_{j+k, \ell+m}^{n} E^{-} u_{j, \ell}^{n}\right) / \tau \\
= & \sum_{(k, m) \in \mathcal{R}_{K}(0,0)} a_{k, m} \sum_{\mathbb{Z}^{2} / \mathcal{R}_{J}(0,0)}\left(E^{+} u_{j+k, \ell+m}^{n} E^{+} u_{j, \ell}^{n}-E^{-} u_{j+k, \ell+m}^{n} E^{-} u_{j, \ell}^{n}\right) / \tau \\
+ & \sum_{(k, m) \in R_{K}(0,0)} a_{k, m}\left(\sum_{\mathbb{Z}^{2} / \mathcal{R}_{J}(0,0)} E^{-} u_{j+k, \ell+m}^{n} E^{+} u_{j, \ell}^{n}-\sum_{\mathbb{Z}^{2} / \mathcal{R}_{J}(-k,-m)} E^{-} u_{j+k, \ell+m}^{n} E^{+} u_{j, \ell}^{n}\right) / \tau \\
= & \sum_{(k, m) \in \mathcal{R}_{K}(0,0)} a_{k, m} \sum_{\mathbb{Z}^{2} / \mathcal{R}_{J}(0,0)}\left(E^{+} u_{j+k, \ell+m}^{n} E^{+} u_{j, \ell}^{n}-E^{-} u_{j+k, \ell+m}^{n} E^{-} u_{j, \ell}^{n}\right) / \tau \\
+ & \sum_{(k, m) \in \mathcal{R}_{K}(0,0)} a_{k, m}\left(\sum_{\mathcal{R}_{J}(-k,-m) / \mathcal{R}_{J}(0,0)} E^{-} u_{j+k, \ell+m}^{n} E^{+} u_{j, \ell}^{n}\right. \\
- & \left.\sum_{\mathcal{R}_{J}(0,0) / \mathcal{R}_{J}(-k,-m)} E_{j+k, \ell+m} E^{+} u_{j, \ell}^{n}\right) / \tau .
\end{aligned}
$$

This leads to

$$
\begin{aligned}
& 2 \sum_{\mathbb{Z}^{2} / \mathcal{R}_{J}(0,0)} \frac{\left|\left(D_{\tau}^{+}\right) u_{j, \ell}^{n}\right|^{2}-\left|\left(D_{\tau}^{-}\right) u_{j, \ell}^{n}\right|^{2}}{\tau} \\
& =\sum_{(k, m) \in \mathcal{R}_{K}(0,0)} a_{k, m} \sum_{\mathbb{Z}^{2} / \mathcal{R}_{J}(0,0)}\left(E^{+} u_{j+k, \ell+m}^{n} E^{+} u_{j, \ell}^{n}-E^{-} u_{j+k, \ell+m}^{n} E^{-} u_{j, \ell}^{n}\right) / \tau \\
& +\frac{1}{\tau} \sum_{(j, \ell) \in \mathcal{B}_{\text {Int }}} \sum_{(k, m) \in \mathcal{B}_{\mathrm{Ext}}} a_{k-j, m-\ell}\left(E^{+} u_{k, m}^{n} E^{-} u_{j, \ell}^{n}-E^{-} u_{k, m}^{n} E^{+} u_{j, \ell}^{n}\right) .
\end{aligned}
$$


Let us now set $\Delta_{k, m}^{E^{+}} u_{j, \ell}^{n}:=E^{+} u_{j+k, \ell+m}^{n}-E^{+} u_{j, \ell}^{n}$. Then, the first term of (27) can be written as

$$
\begin{aligned}
& I_{1}^{n}:=\sum_{(k, m) \in \mathcal{R}_{K}(0,0)} a_{k, m} \sum_{\mathbb{Z}^{2} / \mathcal{R}_{J}(0,0)}\left(E^{+} u_{j+k, \ell+m}^{n} E^{+} u_{j, \ell}^{n}\right) / \tau \\
& =\sum_{(k, m) \neq(0,0)} a_{k, m} \sum_{\mathbb{Z}^{2} / \mathcal{R}_{J}(0,0)}\left(\Delta_{k, m}^{E^{+}} u_{j, \ell}^{n}\right) E^{+} u_{j, \ell}^{n} / \tau \\
& =-\sum_{(k, m) \neq(0,0)} \frac{a_{k, m}}{\tau} \sum_{\mathbb{Z}^{2} / \mathcal{R}_{J}(0,0)}\left(\Delta_{k, m}^{E^{+}} u_{j, \ell}^{n}\right)^{2}+\sum_{(k, m) \neq(0,0)} \frac{a_{k, m}}{\tau} \sum_{\mathbb{Z}^{2} / \mathcal{R}_{J}(0,0)}\left(\Delta_{k, m}^{E^{+}} u_{j, \ell}^{n}\right) E^{+} u_{j+k, \ell+m}^{n} \\
& =-\sum_{(k, m) \neq(0,0)} \frac{a_{k, m}}{\tau} \sum_{\mathbb{Z}^{2} / \mathcal{R}_{J}(0,0)}\left(\Delta_{k, m}^{E^{+}} u_{j, \ell}^{n}\right)^{2}-\sum_{(k, m) \neq(0,0)} \frac{a_{k, m}}{\tau} \sum_{\mathbb{Z}^{2} / \mathcal{R}_{J}(-k,-m)}\left(\Delta_{k, m}^{E^{+}} u_{j, \ell}^{n}\right) E^{+} u_{j, \ell}^{n} \cdot(30)
\end{aligned}
$$

Thus, the term $I_{1}^{n}$ can be written as the average of (29) and (30), i.e.

$$
\begin{aligned}
& I_{1}^{n}=\sum_{(k, m) \neq(0,0)} a_{k, m}\left[\sum_{\mathbb{Z}^{2} / \mathcal{R}_{J}(0,0)}\left(\Delta_{k, m}^{E^{+}} u_{j, \ell}^{n}\right) E^{+} u_{j, \ell}^{n}-\sum_{\mathbb{Z}^{2} / \mathcal{R}_{J}(-k,-m)}\left(\Delta_{k, m}^{E^{+}} u_{j, \ell}^{n}\right) E^{+} u_{j, \ell}^{n}\right] /(2 \tau) \\
& -\sum_{(k, m) \neq(0,0)} a_{k, m} \sum_{\mathbb{Z}^{2} / \mathcal{R}_{J}(0,0)}\left(\Delta_{k, m}^{E^{+}} u_{j, \ell}^{n}\right)^{2} /(2 \tau) .
\end{aligned}
$$

The above equality is equivalent to

$$
\begin{aligned}
& I_{1}^{n}=\sum_{(k, m) \neq(0,0)} a_{k, m}\left[\sum_{\mathcal{R}_{J}(-k,-m) / \mathcal{R}_{J}(0,0)}\left(\Delta_{k, m}^{E^{+}} u_{j, \ell}^{n}\right)\left(-\Delta_{k, m}^{E^{+}} u_{j, \ell}^{n}\right)\right. \\
& \left.-\sum_{\mathcal{R}_{J}(-k,-m) / \mathcal{R}_{J}(0,0)}\left(-\Delta_{k, m}^{E^{+}} u_{j, \ell}^{n}\right) E^{+} u_{j+k, \ell+m}^{n}-\sum_{\mathcal{R}_{J}(0,0) / \mathcal{R}_{J}(-k,-m)}\left(\Delta_{k, m}^{E^{+}} u_{j, \ell}^{n}\right) E^{+} u_{j, \ell}^{n}\right] /(2 \tau) \\
& -\sum_{(k, m) \neq(0,0)} a_{k, m} \sum_{\mathbb{Z}^{2} / \mathcal{R}_{J}(0,0)}\left(\Delta_{k, m}^{E^{+}} u_{j, \ell}^{n}\right)^{2} /(2 \tau) .
\end{aligned}
$$

As a consequence, we have

$$
\begin{aligned}
& I_{1}^{n}=-\sum_{(k, m) \neq(0,0)} a_{k, m} \sum_{\mathcal{R}_{J}(-k,-m) / \mathcal{R}_{J}(0,0)}\left(\Delta_{k, m}^{E^{+}} u_{j, \ell}^{n}\right)^{2} /(2 \tau) \\
& -\sum_{(j, \ell) \in \mathcal{B}_{\text {Int }}} \sum_{(k, m) \in \mathcal{B}_{\text {Ext }}} \frac{a_{k-j, m-\ell}}{\tau} E^{+} u_{j, \ell}^{n}\left(E^{+} u_{k, m}^{n}-E^{+} u_{j, \ell}^{n}\right) \\
& -\sum_{(k, m) \neq(0,0)} \frac{a_{k, m}}{2 \tau} \sum_{\mathbb{Z}^{2} / \mathcal{R}_{J}(0,0)}\left(\Delta_{k, m}^{E^{+}} u_{j, \ell}^{n}\right)^{2} .
\end{aligned}
$$

The term of the right hand side of (31) is equivalent to

$$
-\sum_{(k, m) \neq(0,0)} \frac{a_{k, m}}{2 \tau} \sum_{\mathcal{R}_{J+K}(0,0) / \mathcal{R}_{J}(0,0)}\left(\Delta_{k, m}^{E^{+}} u_{j, \ell}^{n}\right)^{2}
$$

while the term (32) can be written as

$$
-\sum_{(k, m) \neq(0,0)} \frac{a_{k, m}}{2 \tau} \sum_{\mathcal{R}_{J+K}(0,0) / \mathcal{R}_{J}(0,0)}\left(\Delta_{k, m}^{E^{+}} u_{j, \ell}^{n}\right)^{2}-\sum_{(k, m) \neq(0,0)} \frac{a_{k, m}}{2 \tau} \sum_{\mathbb{Z}^{2} / \mathcal{R}_{J+K}(0,0)}\left(\Delta_{k, m}^{E^{+}} u_{j, \ell}^{n}\right)^{2} .
$$


By the above three equality, we deduce

$$
\begin{aligned}
& I_{1}^{n}=-\frac{1}{\tau} \sum_{(j, \ell) \in \mathcal{B}_{\text {Int }}} \sum_{(k, m) \in \mathcal{B}_{\text {Ext }}} a_{k-j, m-\ell}\left[E^{+} u_{j, \ell}^{n}\left(E^{+} u_{k, m}^{n}-E^{+} u_{j, \ell}^{n}\right)+\left(E^{+} u_{k, m}^{n}-E^{+} u_{j, \ell}^{n}\right)^{2}\right] \\
& -\frac{1}{2 \tau} \sum_{(k, m) \neq(0,0)} a_{k, m} \sum_{\mathbb{Z}^{2} / \mathcal{R}_{J+K}(0,0)}\left(\Delta_{k, m}^{E^{+}} u_{j, \ell}^{n}\right)^{2} \\
& =-\frac{1}{\tau} \sum_{(j, \ell) \in \mathcal{B}_{\text {Int }}} \sum_{(k, m) \in \mathcal{B}_{\text {Ext }}} a_{k-j, m-\ell} E^{+} u_{k, m}^{n}\left(E^{+} u_{k, m}^{n}-E^{+} u_{j, \ell}^{n}\right) \\
& -\frac{1}{2 \tau} \sum_{(k, m) \neq(0,0)} a_{k, m} \sum_{\mathbb{Z}^{2} / \mathcal{R}_{J+K}(0,0)}\left(\Delta_{k, m}^{E^{+}} u_{j, \ell}^{n}\right)^{2} \\
& =\frac{1}{\tau} \sum_{(j, \ell) \in \mathcal{B}_{\text {Int }}} \sum_{a_{k, m} \in \mathcal{B}_{\text {Ext }}} a_{k-j, m-\ell} E^{+} u_{k, m}^{n} E^{+} u_{j, \ell}^{n}-H^{n},
\end{aligned}
$$

where we define

$$
H^{n}=\frac{1}{2 \tau} \sum_{(k, m) \neq(0,0)} a_{k, m} \sum_{\mathbb{Z}^{2} / \mathcal{R}_{J+K}(0,0)}\left(\Delta_{k, m}^{E^{+}} u_{j, \ell}^{n}\right)^{2}+\frac{1}{\tau} \sum_{(j, \ell) \in \mathcal{B}_{\text {Int }}} \sum_{(k, m) \in \mathcal{B}_{\text {Ext }}} a_{k-j, m-\ell}\left|E^{+} u_{k, m}^{n}\right|^{2} .
$$

Finally, we can write (27) and (28) as

$$
\begin{aligned}
& 2 \sum_{\mathbb{Z}^{2} / \mathcal{R}_{J}(0,0)} \frac{\left|\left(D_{\tau}^{+}\right) u_{j, \ell}^{n}\right|^{2}-\left|\left(D_{\tau}^{-}\right) u_{j, \ell}^{n}\right|^{2}}{\tau} \\
& =\frac{1}{\tau} \sum_{(j, \ell) \in \mathcal{B}_{\text {Int }}} \sum_{(k, m) \in \mathcal{B}_{\text {Ext }}} a_{k-j, m-\ell}\left(E^{+} u_{k, m}^{n} E^{+} u_{j, \ell}^{n}-E^{-} u_{k, m}^{n} E^{-} u_{j, \ell}^{n}\right) \\
& +\frac{1}{\tau} \sum_{(j, \ell) \in \mathcal{B}_{\text {Int }}} \sum_{(k, m) \in \mathcal{B}_{\mathrm{Ext}}} a_{k-j, m-\ell}\left(E^{+} u_{k, m}^{n} E^{-} u_{j, \ell}^{n}-E^{-} u_{k, m}^{n} E^{+} v_{j, \ell}^{n}\right)-H^{n}+H^{n-1} \\
& =\frac{1}{\tau} \sum_{(j, \ell) \in \mathcal{B}_{\text {Int }}} \sum_{(k, m) \in \mathcal{B}_{\text {Ext }}} a_{k-j, m-\ell}\left(E^{+} u_{k, m}^{n}-E^{-} u_{k, m}^{n}\right)\left(E^{+} u_{j, \ell}^{n}+E^{-} u_{j, \ell}^{n}\right)-H^{n}+H^{n-1} .
\end{aligned}
$$

Let us recall the notations: $(\mathbf{I}[u])^{n}=\left[u_{\nu(1)}^{n}, \cdots, u_{v\left(\left|\mathcal{B}_{\text {Int }}\right|\right)}^{n}\right]^{T},(\mathbf{I}[v])^{n}=\left[v_{\nu(1)}^{n}, \cdots, v_{\nu\left(\left|\mathcal{B}_{\text {Int }}\right|\right)}^{n}\right]^{T}$ and $(\mathbf{E}[w])^{n}=\left[w_{\mu(1)}^{n}, \cdots, w_{\mu\left(\left|\mathcal{B}_{\text {Ext }}\right|\right)}\right]^{T}$. By Theorem 1, we have $(\mathbf{I}[u])^{n}=(\mathbb{A} \star \mathbf{I}[v])^{n}$ and $(\mathbf{E}[u])^{n}=$ $(\mathbb{B} \star \mathbf{I}[v])^{n}$, which implies that: $(\mathbf{E}[u])^{n}=(\mathbb{C} \star \mathbf{I}[u])^{n}$. Summing up (33) from $n=1$ to $N-1$ yields

$$
\begin{aligned}
& 2 \sum_{\mathbb{Z}^{2} / \mathcal{R}_{J}(0,0)} \frac{\left|\left(D_{\tau}^{+}\right) u_{j, \ell}^{N-1}\right|^{2}}{\tau}=2 \sum_{n=1}^{N-1} \sum_{\mathbb{Z}^{2} / \mathcal{R}_{J}(0,0)} \frac{\left|\left(D_{\tau}^{+}\right) u_{j, \ell}^{n}\right|^{2}-\left|\left(D_{\tau}^{-}\right) u_{j, \ell}^{n}\right|^{2}}{\tau} \\
& =\frac{1}{\tau} \sum_{n=1}^{N-1} \sum_{(j, \ell) \in \mathcal{B}_{\text {Int }}} \sum_{(k, m) \in \mathcal{B}_{\text {Ext }}} a_{k-j, m-\ell}\left(E^{+} u_{k, m}^{n}-E^{-} u_{k, m}^{n}\right)\left(E^{+} u_{j, \ell}^{n}+E^{-} u_{j, \ell}^{n}\right)+\sum_{n=1}^{N-1}\left(-H^{n}+H^{n-1}\right) \\
& =\frac{1}{\tau}\left[\sum_{n=1}^{N-1}\left(E^{+}(\mathbb{C} \star \mathbf{I}[u])^{n}-E^{-}(\mathbb{C} \star \mathbf{I}[u])^{n}\right)^{T} \mathbb{S}\left(E^{+}(\mathbf{I}[u])^{n}+E^{-}(\mathbf{I}[u])^{n}\right)\right]-H^{N-1} .
\end{aligned}
$$


Finally, one gets

$$
\begin{aligned}
& \frac{1}{\tau}\left[\sum_{n=1}^{N-1}\left(E^{+}(\mathbb{C} \star \mathbf{I}[u])^{n}-E^{-}(\mathbb{C} \star \mathbf{I}[u])^{n}\right)^{T} \mathbb{S}\left(E^{+}(\mathbf{I}[u])^{n}+E^{-}(\mathbf{I}[u])^{n}\right)\right] \\
& =2 \sum_{\mathbb{Z}^{2} / \mathcal{R}_{J}(0,0)} \frac{\left|\left(D_{\tau}^{+}\right) u_{j, \ell}^{N-1}\right|^{2}}{\tau}+H^{N-1} \geq H^{N-1},
\end{aligned}
$$

which leads to (25).

\subsection{Stability analysis}

We now prove the stability of the fully-discrete CNAC scheme with ABC.

Theorem 2. For $N \tau \leq T$, the solution of the fully discrete CNAC scheme (5) with ABCs (7) satisfies the stability inequality

$$
h^{2} \sum_{\mathcal{R}_{J}(0,0)}\left|u_{j, \ell}^{N}\right|^{2} \leq 2 h^{2} \sum_{\mathcal{R}_{J}(0,0)}\left|u_{j, \ell}^{0}\right|^{2}+2 T^{2} h^{2} \sum_{\mathcal{R}_{J}(0,0)}\left|\left(D_{\tau}^{+}\right) u_{j, \ell}^{0}\right|^{2}
$$

Proof of Theorem 2. Repeating the same procedure as in the proof of Proposition 1 by multiplying (12) by $\left(D_{\tau}^{+}+D_{\tau}^{-}\right) u_{j, \ell}^{n}$, and summing up all the terms for $(j, \ell) \in \mathcal{R}_{J}(0,0)$, we have

$$
\begin{aligned}
& 2 \sum_{\mathcal{R}_{J}(0,0)} \frac{\left|\left(D_{\tau}^{+}\right) u_{j, \ell}^{n}\right|^{2}-\left|\left(D_{\tau}^{-}\right) u_{j, \ell}^{n}\right|^{2}}{\tau} \\
& =\sum_{(k, m) \in \mathcal{R}_{K}(0,0)} a_{k, m} \sum_{\mathcal{R}_{J}(0,0)}\left(E^{+} u_{j+k, \ell+m}^{n} E^{+} u_{j, \ell}^{n}-E^{-} u_{j+k, \ell+m}^{n} E^{-} u_{j, \ell}^{n}\right) / \tau \\
& -\frac{1}{\tau} \sum_{(j, \ell) \in \mathcal{B}_{\text {Int }}} \sum_{(k, m) \in \mathcal{B}_{\mathrm{Ext}}} a_{k-j, m-\ell}\left(E^{+} u_{k, m}^{n} E^{-} u_{j, \ell}^{n}-E^{-} u_{k, m}^{n} E^{+} u_{j, \ell}^{n}\right) .
\end{aligned}
$$

Let us consider the first term of (35) defined by

$$
I_{2}^{n}:=\sum_{(k, m) \in \mathcal{R}_{K}(0,0)} a_{k, m} \sum_{\mathcal{R}_{J}(0,0)}\left(E^{+} u_{j+k, \ell+m}^{n} E^{+} u_{j, \ell}^{n}\right) / \tau .
$$

Then using some similar calculations as for $I_{1}^{n}$ (see (28)), we can prove that

$$
I_{2}^{n}=-G^{n}+\frac{3}{\tau} \sum_{(k, m) \neq(0,0)} a_{k, m} \sum_{\mathcal{R}_{J-K}(0,0)}\left(\Delta_{k, m}^{E^{+}} u_{j, \ell}^{n}\right)^{2},
$$

by defining

$$
\begin{aligned}
& G^{n}=\frac{1}{2 \tau} \sum_{(k, m) \neq(0,0)} a_{k, m} \sum_{\mathcal{R}_{J-K}(0,0)}\left(\Delta_{k, m}^{E^{+}} u_{j, \ell}^{n}\right)^{2}+\frac{1}{\tau} \sum_{(j, \ell) \in \mathcal{B}_{\text {Int }}} \sum_{(k, m) \in \mathcal{B}_{\mathrm{Ext}}} a_{k-j, m-\ell}\left|E^{+} u_{j, \ell}^{n}\right|^{2} \\
& +\frac{2}{\tau} \sum_{(j, \ell) \in \mathcal{B}_{\text {Int }}} \sum_{(k, m) \in \mathcal{B}_{\mathrm{Ext}}} a_{k-j, m-\ell} E^{+} u_{k, m}^{n} E^{+} u_{j, \ell}^{n} .
\end{aligned}
$$


We can rewrite (34) as

$$
\begin{aligned}
& 2 \sum_{\mathcal{R}_{J}(0,0)} \frac{\left|\left(D_{\tau}^{+}\right) u_{j, \ell}^{n}\right|^{2}-\left|\left(D_{\tau}^{-}\right) u_{j, \ell}^{n}\right|^{2}}{\tau} \\
& =\frac{1}{\tau} \sum_{(j, \ell) \in \mathcal{B}_{\text {Int }}} \sum_{(k, m) \in \mathcal{B}_{\mathrm{Ext}}} a_{k-j, m-\ell}\left(-E^{+} u_{k, m}^{n} E^{+} u_{j, \ell}^{n}+E^{-} u_{k, m}^{n} E^{-} u_{j, \ell}^{n}\right) \\
& +\frac{1}{\tau} \sum_{(j, \ell) \in \mathcal{B}_{\text {Int }}} \sum_{(k, m) \in \mathcal{B}_{\mathrm{Ext}}} a_{k-j, m-\ell}\left(-E^{+} u_{k, m}^{n} E^{-} u_{j, \ell}^{n}+E^{-} u_{k, m}^{n} E^{+} u_{j, \ell}^{n}\right)-G^{n}+G^{n-1} \\
& =-\frac{1}{\tau} \sum_{(j, \ell) \in \mathcal{B}_{\text {Int }}} \sum_{(k, m) \in \mathcal{B}_{\mathrm{Ext}}} a_{k-j, m-\ell}\left(E^{+} u_{k, m}^{n}-E^{-} u_{k, m}^{n}\right)\left(E^{+} u_{j, \ell}^{n}+E^{-} u_{j, \ell}^{n}\right)-G^{n}+G^{n-1} .
\end{aligned}
$$

Summing up (36) from $n=0$ to $N-1$, we obtain

$$
\begin{aligned}
& 2 \sum_{\mathcal{R}_{J}(0,0)} \frac{\left|\left(D_{\tau}^{+}\right) u_{j, \ell}^{N-1}\right|^{2}}{\tau}-2 \sum_{\mathcal{R}_{J}(0,0)} \frac{\left|\left(D_{\tau}^{-}\right) u_{j, \ell}^{1}\right|^{2}}{\tau}=2 \sum_{n=1}^{N-1} \sum_{\mathcal{R}_{J}(0,0)} \frac{\left|\left(D_{\tau}^{+}\right) u_{j, \ell}^{n}\right|^{2}-\left|\left(D_{\tau}^{-}\right) u_{j, \ell}^{n}\right|^{2}}{\tau} \\
& =-\frac{1}{\tau} \sum_{n=1}^{N-1} \sum_{(j, \ell) \in \mathcal{B}_{\text {Int }}} \sum_{(k, m) \in \mathcal{B}_{\text {Ext }}} a_{k-j, m-\ell}\left(E^{+} u_{k, m}^{n}-E^{-} u_{k, m}^{n}\right)\left(E^{+} u_{j, \ell}^{n}+E^{-} u_{j, \ell}^{n}\right) \\
& +\sum_{n=1}^{N-1}\left(-G^{n}+G^{n-1}\right) \\
& =-\frac{1}{\tau}\left[\sum_{n=1}^{N-1}\left(E^{+}(\mathbb{C} \star \mathbf{I}[u])^{n}-E^{-}(\mathbb{C} \star \mathbf{I}[u])^{n}\right)^{T} \mathbb{S}\left(E^{+}(\mathbf{I}[u])^{n}+E^{-}(\mathbf{I}[u])^{n}\right)\right]-G^{N-1} \\
& \leq-\frac{1}{\tau} \sum_{(j, \ell) \in \mathcal{B}_{\text {Int }}} \sum_{(k, m) \in \mathcal{B}_{\text {Ext }}} a_{k-j, m-\ell}\left|E^{+} u_{k, m}^{n}\right|^{2}-\frac{1}{\tau} \sum_{(j, \ell) \in \mathcal{B}_{\text {Int }}} \sum_{(k, m) \in \mathcal{B}_{\text {Ext }}} a_{k-j, m-\ell}\left|E^{+} u_{j, \ell}^{n}\right|^{2} \\
& -\frac{2}{\tau} \sum_{(j, \ell) \in \mathcal{B}_{\text {Int }}} \sum_{(k, m) \in \mathcal{B}_{\text {Ext }}} a_{k-j, m-\ell} E^{+} u_{k, m}^{n} E^{+} u_{j, \ell}^{n} \\
& =-\frac{1}{\tau} \sum_{(j, \ell) \in \mathcal{B}_{\text {Int }}} \sum_{(k, m) \in \mathcal{B}_{\text {Ext }}} a_{k-j, m-\ell}\left|E^{+} u_{k, m}^{n}+E^{+} u_{j, \ell}^{n}\right|^{2} .
\end{aligned}
$$

Thus, for any $0 \leq n \leq N-1$, one finally gets

$$
\sum_{\mathcal{R}_{J}(0,0)}\left|\left(D_{\tau}^{+}\right) u_{j, \ell}^{n}\right|^{2} \leq \sum_{\mathcal{R}_{J}(0,0)}\left|\left(D_{\tau}^{+}\right) u_{j, \ell}^{0}\right|^{2},
$$

which leads to

$$
\begin{aligned}
& \sum_{\mathcal{R}_{J}(0,0)}\left|u_{j, \ell}^{N}\right|^{2}=\sum_{R_{J}(0,0)}\left|u_{j, \ell}^{0}+\tau \sum_{n=0}^{N-1}\left(D_{\tau}^{+}\right) u_{j, \ell}^{0}\right|^{2} \leq 2 \sum_{\mathcal{R}_{J}(0,0)}\left|u_{j, \ell}^{0}\right|^{2}+2 \sum_{R_{J}(0,0)}\left|\tau \sum_{n=0}^{N-1}\left(D_{\tau}^{+}\right) u_{j, \ell}^{0}\right|^{2} \\
& \leq 2 \sum_{\mathcal{R}_{J}(0,0)}\left|u_{j, \ell}^{0}\right|^{2}+2 N \tau^{2} \sum_{\mathcal{R}_{J}(0,0)} \sum_{n=0}^{N-1}\left|\left(D_{\tau}^{+}\right) u_{j, \ell}^{0}\right|^{2} \leq 2 \sum_{\mathcal{R}_{J}(0,0)}\left|u_{j, \ell}^{0}\right|^{2}+2 T \tau N \sum_{\mathcal{R}_{J}(0,0)}\left|\left(D_{\tau}^{+}\right) u_{j, \ell}^{0}\right|^{2} \\
& \leq 2 \sum_{\mathcal{R}_{J}(0,0)}\left|u_{j, \ell}^{0}\right|^{2}+2 T^{2} \sum_{\mathcal{R}_{J}(0,0)}\left|\left(D_{\tau}^{+}\right) u_{j, \ell}^{0}\right|^{2} .
\end{aligned}
$$


By the RK4 scheme $(24),\left(D_{\tau}^{+}\right) u_{j, \ell}^{0}$ is bounded for small $\tau$ (at least with third order of accuracy for approximating $\left.\partial_{t} u_{j, \ell}^{0}\right)$. Therefore, the term $\sum_{\mathcal{R}_{J}(0,0)}\left|\left(D_{\tau}^{+}\right) u_{j, \ell}^{0}\right|^{2}$ in the above inequality is also bounded when $\tau$ is small, ending hence the proof of Theorem 2 .

\section{Numerical example}

We consider the nonlocal wave equation (1) with the interaction kernel function $\gamma_{\delta}$ given by

$$
\gamma_{\delta}(r)=\frac{6}{r \pi \delta^{3}}, \quad 0 \leq r:=\sqrt{x^{2}+y^{2}} \leq \delta,
$$

for three possible initial data

$$
\phi_{1}^{0}(x, y, 0)=e^{-8 r^{2}} J_{0}\left(\frac{r}{2}\right), \quad \phi_{2}^{0}(x, y, 0)=(x+y) \frac{e^{-4 r^{2}}}{r} J_{1}\left(\frac{r}{2}\right), \quad \phi_{3}^{0}(x, y, 0)=700 x y \frac{e^{-4 r^{2}}}{r} J_{2}\left(\frac{r}{2}\right),
$$

where $J_{m}$ is the Bessel's function of order $m$. In addition, we fix $\phi_{j}^{1}=0$, for $j=1,2,3$. The computational domain is set as $[-2,2]^{2}$ and the final computational time is $T=5$. The reference solutions $u^{\text {ref }}$ are computed on the larger spatial domain $[-16,16]^{2}$ to avoid any boundary effect, for the small discretization parameters $h=2^{-9}$ and $\tau=2^{-12}$. For $\delta=0.125$ and $\delta=0.25$, and for the three initial data, we report in Table 1 the $L^{\infty}$-norm error $e_{\infty, T}^{h, \tau}$ defined by

$$
e_{\infty, T}^{h, \tau}=\max _{-J \leq j, \ell \leq J}\left|u^{\mathrm{ref}}\left(x_{j}, y_{\ell}, T\right)-u_{j, \ell}^{N}\right|
$$

by refining $h$, and fixing $\tau=2^{-12}$ and for $T=5$. In addition, Figure 3 shows the behaviour of the numerical solution with initial data $\phi_{2}^{0}$ (and $\phi_{2}^{1}=0$ ) at different times for $\delta=0.25, h=2^{-7}$ and $\tau=2^{-12}$. We observe that the wave packet goes through the corners without any reflection back.

\begin{tabular}{|c||c||c|c|c|c|c|}
\hline Initial data & $\delta$ & $h=2^{-4}$ & $h=2^{-5}$ & $h=2^{-6}$ & $h=2^{-7}$ & cvg. rate \\
\hline \hline$\phi_{1}^{0}$ & 0.125 & $1.31 \times 10^{-3}$ & $3.58 \times 10^{-4}$ & $5.79 \times 10^{-5}$ & $1.21 \times 10^{-5}$ & 2.29 \\
\hline$\phi_{1}^{0}$ & 0.25 & $3.74 \times 10^{-4}$ & $7.44 \times 10^{-5}$ & $1.20 \times 10^{-5}$ & $2.17 \times 10^{-6}$ & 2.39 \\
\hline \hline$\phi_{2}^{0}$ & 0.125 & $7.15 \times 10^{-4}$ & $2.05 \times 10^{-4}$ & $3.38 \times 10^{-5}$ & $6.96 \times 10^{-6}$ & 2.26 \\
\hline$\phi_{2}^{0}$ & 0.25 & $6.67 \times 10^{-4}$ & $1.36 \times 10^{-4}$ & $2.51 \times 10^{-5}$ & $6.60 \times 10^{-6}$ & 2.24 \\
\hline \hline$\phi_{3}^{0}$ & 0.125 & $9.95 \times 10^{-4}$ & $3.27 \times 10^{-4}$ & $1.68 \times 10^{-5}$ & $3.37 \times 10^{-6}$ & 2.24 \\
\hline$\phi_{3}^{0}$ & 0.25 & $1.15 \times 10^{-3}$ & $2.47 \times 10^{-4}$ & $1.37 \times 10^{-4}$ & $1.77 \times 10^{-5}$ & 1.89 \\
\hline
\end{tabular}

Table 1: Evolution of the error $e_{\infty, T}^{h, \tau}$ vs $h$ for the three initial data $\phi_{j}^{0}, j=1,2,3$ (for $\tau=2^{-12}$ and $T=5$ ).

\section{Conclusion}

The construction of ABCs is proposed for the two-dimensional peridynamics equation which is fully discretized by the Crank-Nicolson scheme in time and an asymptotically compatible scheme in space. A recursive algorithm is built for evaluating numerically the Green's functions involved in the expression of the ABCs for the fully discretized peridynamics. In addition, the stability of the scheme is stated. Numerical examples confirm that the ABCs are accurate. In particular, we show that that there is no spurious reflection at the corners of the square domain. Further works include the error analysis as well as considering nonlinearities into the peridynamics equation. 

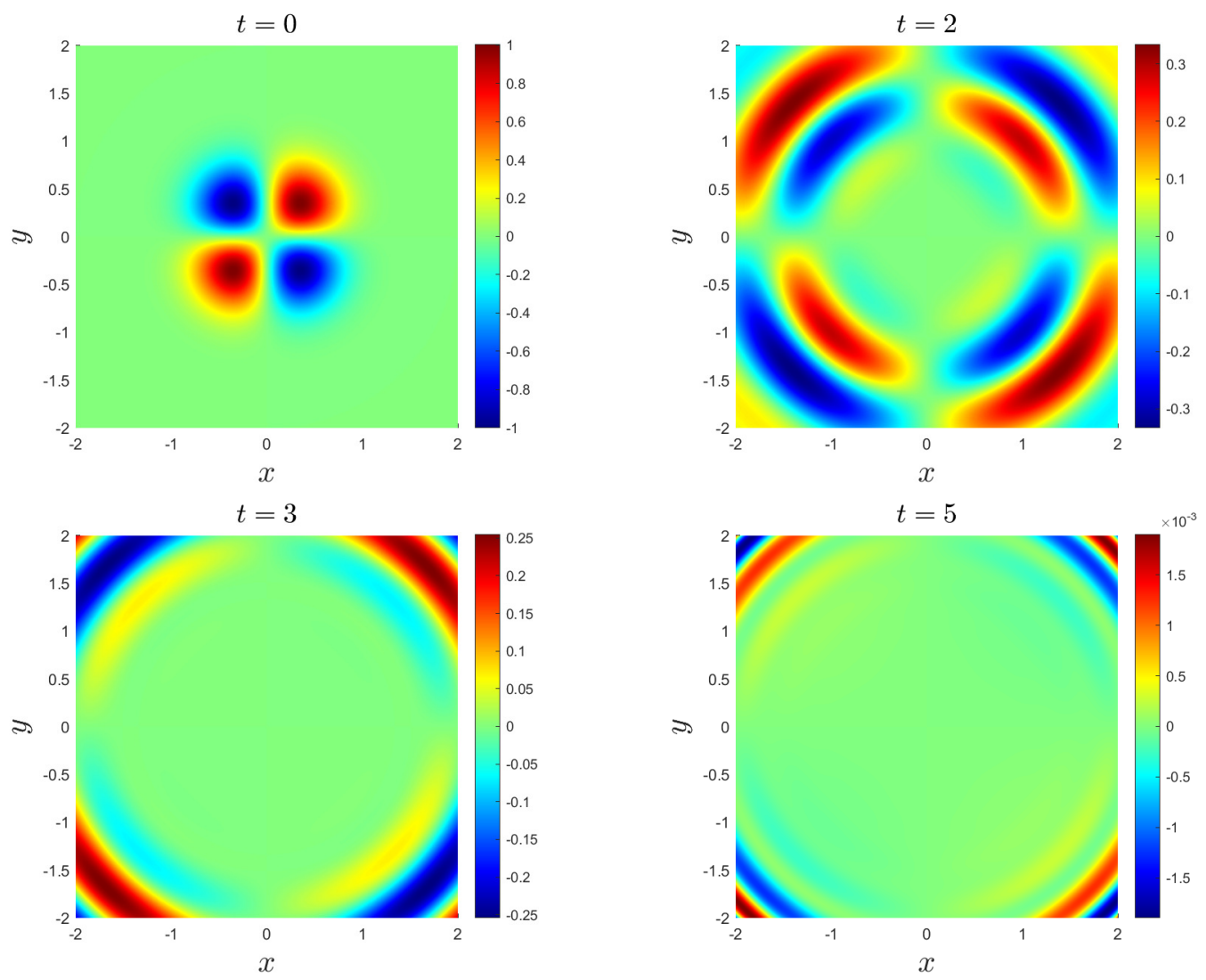

Figure 3: Snapshots of the numerical solution $|u|$ at times $t=0,2,3,5\left(\delta=0.25, h=2^{-7}, \tau=2^{-12}\right)$.

\section{Acknowledgments}

This research is partially supported by NSFC under grant Nos. 11832001, 11502028. Research conducted within the context of the Sino-French International Associated Laboratory for Applied Mathematics-LIASFMA. X. ANTOINE thanks the LIASFMA funding support of the Université de Lorraine.

\section{References}

[1] X. Antoine, A. Arnold, C. Besse, M. Ehrhardt, A. Schädle, A review of transparent and artificial boundary conditions techniques for linear and nonlinear Schrödinger equations, Communications in Computational Physics 4 (4) (2008), pp. 729-796.

[2] X. Antoine and E. Lorin, Towards Perfectly Matched Layers for time-dependent space fractional PDEs, Journal of Computational Physics, 391, (2019), pp. 59-90.

[3] X. Antoine, E. Lorin and Q. Tang, A friendly review of absorbing boundary conditions and perfectly matched layers for classical and relativistic quantum waves equations, Molecular Physics, 115 (15-16), (2017), pp. 1861-1879.

[4] X. Antoine, E. Lorin and Y. Zhang, Derivation and analysis of computational methods for fractional Laplacian equations with absorbing layers, Numerical Algorithms, 87, (2021), pp. 409444 . 
[5] B. Baeumer, M. Kovcs, M.M. Meerschaert, and H. Sankaranarayanan, Boundary conditions for fractional diffusion, Journal of Computational and Applied Mathematics, 336, (2018), pp.408424.

[6] A. Bayliss and E. Turkel, Radiation boundary conditions for wave-like equations, Communications on Pure and Applied Mathematics, 33(6), (1981), pp. 707-725.

[7] C. Bekar and E. Madenci, Peridynamics enabled learning partial differential equations, Journal of Computational Physics, 434, (2021), 110193.

[8] M. D'Elia, Q. Du, C. Glusz, M. Gunzburger, X. Tian and Z. Zhou, Numerical methods for nonlocal and fractional models, Acta Numerica, 29, (2020), pp.1-124.

[9] Q. Du, Nonlocal Modelling, Analysis and Computation, CBMS-NSF Regional Conference Series in Applied Mathematics, 94, SIAM, 2019.

[10] Q. Du, H. Han, J. Zhang and C. Zheng, Numerical solution of a two-dimensional nonlocal wave equation on unbounded domains, SIAM Journal on Scientific Computing, 40, (2018), pp.14301445 .

[11] Q. Du, Y. Tao and X. Tian, Asymptotically compatible discretization of multidimensional nonlocal diffusion models and approximation of nonlocal Green's functions, IMA Journal of Numerical Analysis, 39, (2019), pp.607-625.

[12] Q. Du, J. Zhang and C. Zheng, Nonlocal wave propagation in unbounded multiscale media, Communications in Computational Physics, 24, (2018), pp.1049-1072.

[13] B. Engquist and A. Majda, Absorbing boundary conditions for the numerical simulation of waves, Mathematics of Computation, 31(139), (1977), pp.629-651.

[14] B. Engquist and A. Majda, Radiation boundary conditions for acoustic and elastic calculations, Communications on Pure and Applied Mathematics, 32, (1979), pp.313-357.

[15] W. Gerstle, N. Sau and S. Silling, Peridynamic modeling of concrete structures, Nuclear Engineering and Design, 237, (2007), pp.1250-1258.

[16] D. Givoli, High-order local non-reflecting boundary conditions: a review, Wave Motion, 39(4), (2004), pp.319-326.

[17] T. Hagstrom, Radiation boundary conditions for the numerical simulation of waves, Acta Numerica, 8, (1999), pp. 47-106.

[18] H. Han and X. Wu, Artificial Boundary Method. Spring-Verlag and Tsinghua University Press, 2013.

[19] S. Ji, Y. Yang, G. Pang and X. Antoine, Accurate artificial boundary conditions for the semidiscretized linear Schrödinger and heat equations on rectangular domains, Computer Physics Communications, 222, (2018) pp.84-93.

[20] J.F. Kelly, H. Sankaranarayanan and M.M. Meerschaert, Boundary conditions for two-sided fractional diffusion, Journal of Computational Physics, 376, (2019), pp.1089-1107.

[21] B. Kilic, A. Agwai and E. Madenci, Peridynamic theory for progressive damage prediction in center-cracked composite laminates, Composite Structures, 90, (2009), pp.141-151. 
[22] E. Madenci, A. Barut and M. Dorduncu, Peridynamic Differential Operators for Numerical Analysis, Springer, Boston, MA, 2019.

[23] E. Madenci, A. Barut, M. Dorduncu and M. Futch, Numerical solution of linear and nonlinear partial differential equations by using the peridynamic differential operator, Numerical Methods for Partial Differential Equations, 33, (2017), pp.1726-1753.

[24] E. Madenci, A. Barut and M. Futch, Peridynamic differential operator and its applications, Computer Methods in Applied Mechanics and Engineering, 304, (2016), pp.408-451.

[25] Y. Mikata, Analytical solutions of peristatic and peridynamic problems for a $1 D$ infinite rod, International Journal of Solids and Structures, 49, (2012), pp.2887-2897.

[26] E. Oterkus and E. Madenci, Peridynamic analysis of fiber-reinforced composite materials, Journal of Mechanics of Materials and Structures, 70, (2012), pp.45-84.

[27] E. Oterkus and E. Madenci, Peridynamic theory for damage initiation and growth in composite laminate, Advances in Fracture and Damage Mechanics, 488, (2012), pp.355-358.

[28] G. Pang, S. Ji and X. Antoine, Artificial boundary conditions for the semi-discretized onedimensional nonlocal Schrödinger equation, Journal of Computational Physics, 444, (2021), 110575 .

[29] G. Pang, S. Ji, Y. Yang and S. Tang, Eliminating corner effects in square lattice simulation, Computational Mechanics, 62, (2018), pp.111-122.

[30] G. Pang, Y. Yang, X. Antoine and S. Tang, Stability and convergence analysis of artificial boundary conditions for the Schrödinger equation on a rectangular domain, Mathematics of Computation, 90, (2021), pp. 2731-2756.

[31] S. Silling, Reformulation of elasticity theory for discontinuities and long-range forces, Journal of the Mechanics and Physics of Solids, 48, (2000), pp.175-209.

[32] S. Tang, S. Zhu, D. Qian, Energy-based matching boundary conditions for non-ordinary peridynamics in one space dimension, International Journal for Multiscale Computation Engineering, 16, (2020), pp.611-636.

[33] S.V. Tsynkov, Numerical solution of problems on unbounded domains. A review, Applied Numerical Mathematics, 27(4), (1998), pp.465-532.

[34] J. Wang, J. Zhang, C. Zheng, Stability and error analysis for a second-order approximate of the $1 D$ nonlocal Schrödinger equation under DtN-type boundary conditions, to appear.

[35] L. Wang, Y. Chen, J. Xu, and J. Wang, Transmitting boundary conditions for $1 D$ peridynamics, International Journal of Numerical Methods in Engineering, 110, (2017), pp. 379-400.

[36] O. Weckner and R. Abeyaratne, The effect of long-range forces on the dynamics of a bar, Journal of the Mechanics and Physics of Solids, 53, (2005), pp.705-728.

[37] O. Weckner and E. Emmrich, Numerical simulation of the dynamics of a nonlocal, inhomogeneous, infinite bar, Journal of Computational and Applied Mechanics, 6, (2005), pp.311-319.

[38] J. Xu, A. Askari, O. Weckner and S. Silling, Peridynamic analysis of impact damage in composite laminates, Journal of Aerospace Engineering, 21, (2008), pp.187-194. 
[39] Y. Yan, J. Zhang, and C. Zheng, Numerical computations of nonlocal Schrödinger equations on the real line, Communications on Applied Mathematics and Computation, 2, (2020), pp.241-260.

[40] W. Zhang, J. Yang, J. Zhang, and Q. Du, Absorbing boundary conditions for nonlocal heat equations on unbounded domain, Communications in Computational Physics, 21 (2017), pp.1639.

[41] C. Zheng, J. Hu, Q. Du and J. Zhang, Numerical solution of the nonlocal diffusion equation on the real line, SIAM Journal on Scientific Computing, 39, (2017), pp.1951-1968. 